\title{
Methods for the combination of kernel matrices within a support vector framework
}

\author{
Isaac Martín de Diego • Alberto Muñoz • \\ Javier M. Moguerza
}

Received: 1 March 2006 / Revised: 7 April 2009 / Accepted: 5 July 2009 / Published online: 14 August 2009 Springer Science+Business Media, LLC 2009

\begin{abstract}
The problem of combining different sources of information arises in several situations, for instance, the classification of data with asymmetric similarity matrices or the construction of an optimal classifier from a collection of kernels. Often, each source of information can be expressed as a similarity matrix. In this paper we propose a new class of methods in order to produce, for classification purposes, a single kernel matrix from a collection of kernel (similarity) matrices. Then, the constructed kernel matrix is used to train a Support Vector Machine (SVM). The key ideas within the kernel construction are twofold: the quantification, relative to the classification labels, of the difference of information among the similarities; and the extension of the concept of linear combination of similarity matrices to the concept of functional combination of similarity matrices. The proposed methods have been successfully evaluated and compared with other powerful classifiers and kernel combination techniques on a variety of artificial and real classification problems.
\end{abstract}

Keywords Kernel methods · Combination of kernels · Similarity-based classification · Support Vector Machines

\section{Introduction}

Support Vector Machines (SVMs) have proven to be a successful tool for the solution of a wide range of classification problems since their introduction in Cortes and Vapnik (1995).

Editor: Dale Schuurmans.

I.M. de Diego (凶) · J.M. Moguerza

University Rey Juan Carlos, c/ Tulipán s/n, 28933 Móstoles, Spain

e-mail: isaac.martin@urjc.es

J.M. Moguerza

e-mail: javier.moguerza@urjc.es

\section{A. Muñoz}

University Carlos III, c/ Madrid 126, 28903 Getafe, Spain

e-mail: alberto.munoz@uc3m.es 
The method uses as a primary source of information a kernel matrix $K$, whose entries $K_{i j}$ are calculated by using a Mercer's kernel (i.e. a positive definite function). Roughly speaking, a kernel $K(x, z)$ is a real-valued function $K: X \times X \rightarrow \mathbb{R}$ such that there exists a function $\Phi: X \rightarrow Z$, where $Z$ is a real vector space, with the property $K(x, z)=\Phi(x)^{T} \Phi(z)$. The kernel $K(x, z)$ acts as a dot product in the space $Z$. in the sample. $X$ and $Z$ are called, respectively, input space and feature space. SVM classifiers construct a maximum margin hyperplane in the feature space. By the representer theorem (see, for instance, Schölkopf et al. 2000), the SVM decision hyperplane always takes the form $f(x)=\sum_{i} \alpha_{i} y_{i} K\left(x, x_{i}\right)+b$, where $\left\{\left(x_{i}, y_{i}\right) \in X \times Y\right\}_{i=1}^{l}$ is a fixed training data sample with $Y=\{-1,+1\}$. In the following, when the context is clear, we will notate both, the kernel function and the matrix derived from the kernel function, as $K$. The approximation and generalization capacity of the SVM is determined by the choice of $K$ (Cristianini and Shawe-Taylor 2000). However, it is hard to know in advance which particular kernel function has to be used for a given data set. Fortunately, as we will show, kernels are related to similarity measures (see Burges 1998), and this information is actually available in many data analysis problems. In addition, working with similarities avoids the need to explicitly work with Euclidean coordinates. This is particularly useful for data sets involving strings, trees, microarrays or text data sets, for instance. Nevertheless, using a single similarity measure may not be enough to solve accurately the problem under consideration. This happens, for instance, when dealing with text mining problems, where analysis results may vary depending on the document similarity measure chosen (Joachims 2002). Thus, information provided by a single similarity measure may not be enough for classification purposes, and the combination of similarities appears as an interesting alternative to the choice of the 'best' similarity.

The specific literature on the combination of matrix-like sources of information is rather in its beginnings. For the particular case of information arising from kernel matrices, a usual approach is to consider linear combinations of the matrices. This is the proposal in Lanckriet et al. (2004), which is based on the solution of a semi-definite programming problem (see Vandenberghe and Boyd 1996) to calculate the coefficients of the linear combination. In Bach et al. (2004) and Bousquet and Herrmann (2003) special-purpose implementations, in order to improve the computational cost required for the solution of this type of optimization problems, are supplied. The main difference between both approaches is the way in which the weights within the semi-definite programming problem are found. The ideas introduced by Lanckriet et al. (2004) are extended in Ong et al. (2005). This work is based on the definition of a kernel (called hyperkernel) in the space of kernels itself, leading to the semidefinite optimization problem. Finally, it is worthwhile to mention the proposal in Bennett et al. (2002). The method, called MARK-L, builds a classifier (not the specific kernel matrix) by a boosting type algorithm.

In this paper, we provide a general framework to build, for classification purposes, a kernel matrix from a collection of similarity matrices. This framework includes and extends the very preliminary works in Moguerza et al. (2004), and in de Diego et al. (2004): a unifying framework is proposed, theoretical results are shown and conclusive numerical results are given. Two general schemes for combining the available similarity matrices are developed. The first one is based on the quantification of the difference of information among the similarities. The second is based on the concept of functional combination of similarity matrices, which extends the concept of linear combination of similarities. In both cases the label information is used to build the final kernel matrix. These schemes differ from the ones already existing in the literature. As a first contribution, we are able to work with similarity matrices, which constitute a more general class, including the one made up by kernel matrices. Another and perhaps more important contribution is the possibility of combining 
different sources of information in a non-linear manner. The key idea of this non-linear approach is the inclusion of the label information in the process of building the final kernel matrix. In this way, we are able to exploit the structure of the joint space where the data and their corresponding labels live, that is, the feature space embeds both the input and the output. Out of the scope of the combination of different sources of information, there are some previous proposals involving the use of this kind of feature space (Kwok and Tsang 2003; Tsochantaridis et al. 2004). From these two works, the only one that explicitly uses a joint input/output feature space is the proposal by Tsochantaridis et al. (2004). Their method is based on the use of a feature map over input/output pairs, so that different values for the kernel matrix are possible assuming different labels for the inputs. Then, based on the different values of the kernel, a special SVM classification problem is designed.

The paper is organized as follows. The general framework is presented in Sect. 2. In Sect. 3, the problem at hand is motivated. Section 4 describes the proposed method for combining kernel matrices using the difference of information among kernels. Section 5 describes the proposed method based on the functional combination of kernel matrices. The experimental setup and results on artificial and real data sets are resumed in Sect. 6. Section 7 concludes. Appendix A describes in detail all the experiments. Appendix B contains the proof of a proposition in Sect. 4.4.

\section{General framework}

For our purposes, a similarity (dissimilarity) measure is a function that associates a numeric value with a pair of objects with the idea that a higher value indicates greater likeness (unlikeness) between the objects. There is an explicit relation between a kernel matrix and a distance-based similarity matrix. For instance, consider a matrix $K$ of inner products in an Euclidean space $\mathcal{F}$ (a kernel). Then $D^{2}=k 1_{l}^{T}+1_{l} k^{T}-2 K$ is a matrix of square Euclidean distances in $\mathcal{F}$ (Gower and Legendre 1986), where $k$ is a column vector made up of the diagonal elements of $K$, and $1_{l}$ is a $l$-dimensional column vector of ones. It is well known that every distance is a dissimilarity measure, and several methods to transform dissimilarities into similarities have been proposed in the literature (see, for instance, Pȩkalska and Duin 2005, for a complete review). Therefore, in the following we will work with similarities. This is not a constraint since, given a kernel matrix and using the previous arguments, it is immediate to build a similarity matrix. The general framework that we will follow within this work is shown in Algorithms 1 and 2, which correspond respectively to the training and testing phases of the classification task.

\subsection{Training algorithm}

Given a labeled training data set $\left\{\left(x_{1}, y_{1}\right), \ldots,\left(x_{l}, y_{l}\right)\right\}$, the following algorithm describes how to calculate the weights that will be used at testing time to build a discrimination rule.

The normalization referred to in Step 1 is performed in order to make the kernel matrices comparable in magnitude. With this aim, the entry $(i, j)$ of the normalized matrix will take the value: $\left(K_{i j}-K_{\min }\right) /\left(K_{\max }-K_{\min }\right)$, where $K_{\min }$ and $K_{\max }$ are, respectively, the overall minimum and maximum of the matrix $K$. Another possibility is to normalize the entries as in Shawe-Taylor and Cristianini (2004), that is, the entry $(i, j)$ of the normalized matrix will take the value: $K_{i j} / \sqrt{K_{i i} K_{j j}}$.

The aim of this paper is to develop methods to build the matrix $K^{*}$ in Step 2. Sections 3, 4 and 5 are devoted to describe these methods. Regarding Step 3, building a symmetric positive semi-definite (psd) matrix from a similarity matrix is only straightforward for Euclidean 


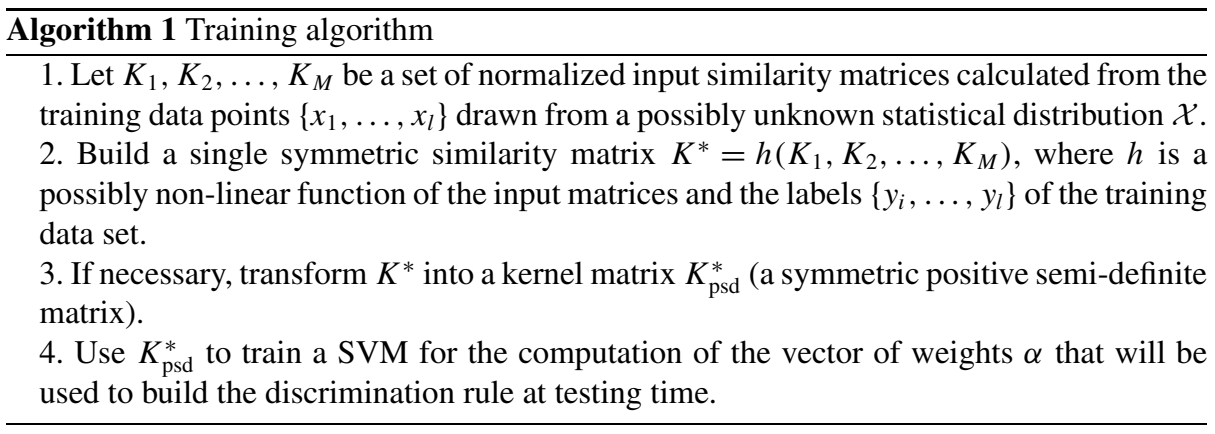

distance-based similarities (Burges 1998). In Sect. 2.3 we will motivate and develop this step of Algorithm 1.

\subsection{Testing algorithm}

Given an unlabeled data point $x, K_{\mathrm{psd}}^{*}\left(x, x_{i}\right)$ has to be evaluated. Since labels are needed to evaluate $K^{*}$ (and therefore $K_{\mathrm{psd}}^{*}$ ), we can calculate two different values for $K_{\mathrm{psd}}^{*}\left(x, x_{i}\right)$ : the first one, $K_{+}^{*}\left(x, x_{i}\right)$, assuming $x$ belongs to class +1 , and the second, $K_{-}^{*}\left(x, x_{i}\right)$, assuming $x$ belongs to class -1 . For each assumption, all we have to do is to predict the class $x$ belongs to. This can be made by calculating the conditional decision hyperplanes under each assumption, that is $f_{+}(x)$ and $f_{-}(x)$. Then, using a voting scheme, the a posteriori class for $x$ can be predicted. Algorithm 2 summarizes these stages.

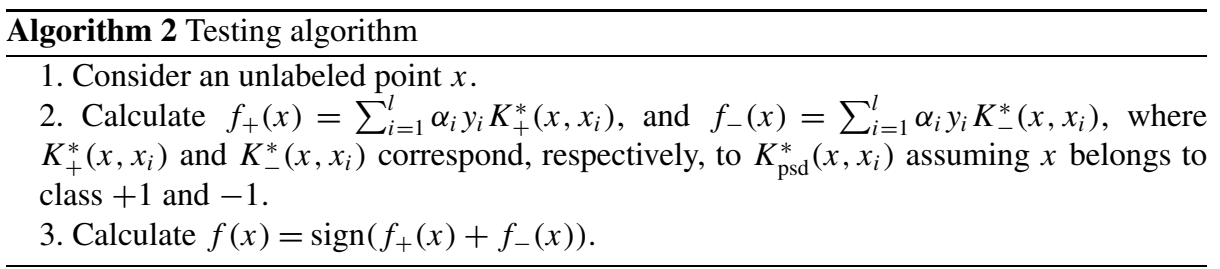

It is interesting to remark that, if $\operatorname{sign}\left(f_{+}(x)\right)=\operatorname{sign}\left(f_{-}(x)\right)$, then the a priori assumption on the class of $x$ is not relevant on the a posteriori classification of $x$. Regarding points such that $\operatorname{sign}\left(f_{+}(x)\right) \neq \operatorname{sign}\left(f_{-}(x)\right)$, they are assigned to the class corresponding to the largest distance from each conditional discrimination hyperplane. We will refer to these points as voting discrepancies. Being rigorous, the voting discrepancies should be unlabeled, that is, the class they belong to can not be determined. In a standard support vector framework, this is similar to the situation that occurs with points that geometrically lie within the margin (known as margin errors, see Schölkopf and Smola 2002). Nevertheless, in the literature, it is accepted to classify the margin errors into the class corresponding to the side of the hyperplane where they lie. Since for test-data no labels are available, this is a natural way to proceed. A similar idea is followed in Tsochantaridis et al. (2004), where the margin is maximized subject to the following constraint: for each point, the distance from the hyperplane to the representation corresponding to the true label has to be larger than the distance from the hyperplane to the representation corresponding to other labels. In our approach, the definition of $K^{*}$ in the training phase will maximize both, the dissimilarities among points 
belonging to different classes and the similarities among points belonging to the same class, which implicitly increases the margin. In the testing phase, based on the discrimination function built from $K^{*}$, points are assigned to classes by choosing the label that maximizes its distance to the hyperplane.

In Sect. A.2.1 (in Appendix A), we will show that there is no risk in using this approach, in the sense that, when the similarities are coherent with the classification labels (that is, they have been appropriately defined to solve the classification problem), the expected situation $\operatorname{should}$ be $\operatorname{sign}\left(f_{+}(x)\right)=\operatorname{sign}\left(f_{-}(x)\right)$.

\subsection{Building positive semi-definite matrices}

Schemes based on the one proposed in Step 2 of Algorithm 1 may lead to indefinite matrices $K^{*}$. However, in order to make this methodology useful for many classification techniques (including SVMs), $K^{*}$ should be a positive semi-definite symmetric matrix. Several techniques have been proposed to solve this problem (see, for a complete description, Pȩkalska et al. 2001). But there seems not to be a universally best method (Pȩkalska et al. 2004). We will describe how these techniques are applied to build a positive semidefinite matrix $K_{\mathrm{psd}}^{*}$ that will be used at training time. In addition, we will show that at testing time $K_{\mathrm{psd}}^{*}$ is well defined, that is, its expression for training data when using them at testing time does not change. Recently, SVM classifiers with non-positive definite matrices have been discussed (Haasdonk 2005; Ong et al. 2004), but further analysis is required.

In the following we will denote the training data as $\left(x_{1}, \ldots, x_{l}\right)$, and the vector of kernel values for any point $x$ with respect to this training set as $K_{x}^{*}=\left(K^{*}\left(x, x_{1}\right), \ldots, K^{*}\left(x, x_{l}\right)\right)^{T}$.

\subsubsection{Second power of the kernel matrix}

The first possibility we will consider is a representation of data points leading to the kernel matrix $K_{\mathrm{psd}}^{*}=K^{*} K^{*}$, that is, the second power of the kernel matrix. Consider the following representation for any point $x$ :

$$
z_{x}=K_{x}^{*},
$$

that is, the vector of kernel values for $x$ with respect to the training data set. Given any pair of points $(u, v)$, we can define the kernel $K_{\mathrm{psd}}^{*}(u, v)$ as:

$$
K_{\mathrm{psd}}^{*}(u, v)=z_{u}^{T} z_{v}
$$

Notice that the expression of $z_{x}$ does not change at testing time for points in the training set, that is the kernel is well defined. This new kernel $K_{\text {psd }}^{*}$ is the one that we will use at training and testing time. Using this representation, the kernel matrix for the training set is $K^{*} K^{*}$. Consider the spectral decomposition $K^{*}=Q \Lambda Q^{T}$, where $\Lambda$ is a diagonal matrix containing the eigenvalues of $K^{*}$, and $Q$ is an orthonormal matrix whose columns are the corresponding eigenvectors. It holds that $K_{\mathrm{psd}}^{*}=Q \Lambda Q^{T} Q \Lambda Q^{T}=Q \Lambda^{2} Q^{T}$, a psd matrix. It is important to remark that the representation $z_{x}$ is calculated (for every data point) using always the training data set, that is, we are defining a kernel whose expression in closed-form depends on a fixed data sample (the training set, see for instance, Muñoz and Moguerza 2006). From a computational point of view, this technique is very cheap in practice as it only requires a product of two matrices and it is advisable when the size of the kernel matrix involved is large. 


\subsubsection{Multidimensional scaling}

Another direct approach uses Multidimensional Scaling (MDS) to represent the data set in a Euclidean space. Consider the spectral decomposition $K^{*}=Q \Lambda Q^{T}$, where $\Lambda$ is a diagonal matrix containing (in decreasing order) the eigenvalues of $K^{*}$, and $Q$ is an orthonormal matrix whose columns are the corresponding eigenvectors. Assume that $\Lambda$ has at least $p$ positive eigenvalues and consider $Q_{p}$, a matrix whose columns are the first $p$ eigenvectors. Given a point $x$, its $p$-dimensional representation is:

$$
z_{x}=\Lambda_{p}^{-\frac{1}{2}} Q_{p}^{T} K_{x}^{*}
$$

Given any pair of points $(u, v)$, we define the kernel $K_{\mathrm{psd}}^{*}(u, v)$ as:

$$
K_{\mathrm{psd}}^{*}(u, v)=z_{u}^{T} z_{v} .
$$

Again, we are defining a kernel whose expression in closed-form depends on a fixed data sample and with the same properties of the kernel defined in the previous section. As before, the kernel is well defined as the expression of $z_{x}$ does not change at testing time for training points. It can be shown (Pȩkalska et al. 2001) that for the training data $K_{\mathrm{psd}}^{*}=Q_{p} \Lambda_{p} Q_{p}^{T}$, a psd matrix by construction. Given that the spectral decomposition is needed, this technique is more expensive than the one in Sect. 2.3.1. Nevertheless, it is the most popular in the literature, and we include it for the sake of completeness. In fact, this method can be viewed as a Principal Component Analysis (see, for instance, Cristianini and Shawe-Taylor 2000; Cox and Cox 1994).

\section{Building a single symmetric similarity matrix}

In the following, we will concentrate in Step 2 of Algorithm 1, that is, the construction of a single symmetric similarity matrix from a set of similarity matrices. We will begin with a particular case of the problem at hand: the classification of data whose similarity matrix is asymmetric. Because of its simplicity, this case allows the motivation of the main sections in this work. Examples of such matrices arise when considering citations among journals or authors, sociometric data (Zielman and Heiser 1996), or word association strengths (Muñoz 1997). In the first case, suppose a paper (Web page) $x_{i}$ cites (links to) a paper (Web page) $x_{j}$, but the opposite is not true. In the second example, a child $x_{i}$ may select another child $x_{j}$ to sit next in their classroom, but not reciprocally. In the third case, word $x_{i}$ may appear in documents where word $x_{j}$ occurs, but not conversely. To be more specific, let $S$ be an asymmetric $l \times l$ similarity matrix (corresponding to a data set of $l$ individuals), that is, $s_{i j} \neq s_{j i}$. In all these cases, each triangular part of the similarity matrix should be considered as a different source of information. Usually, symmetrization is achieved by defining the elements in the symmetric matrix as $K^{*}\left(x_{i}, x_{j}\right)=\frac{s_{i j}+s_{j i}}{2}$, that is, $K^{*}=\frac{1}{2}\left(S+S^{T}\right)$. In this way, the same importance is given to $s_{i j}$ and $s_{j i}$, the two sources of information. This symmetrization method is related to the decomposition of a matrix into its symmetric and skew-symmetric parts:

$$
S=\frac{1}{2}\left(S+S^{T}\right)+\frac{1}{2}\left(S-S^{T}\right) .
$$

In particular, the skew-symmetric part $\frac{1}{2}\left(S-S^{T}\right)$ is being ignored, which implies a loss of information. Other methods to obtain a symmetric matrix have been proposed (Schölkopf 
et al. 1999; Tsuda 1999) based on considering $K^{*}=S^{T} S$ and $K^{*}=S S^{T}$. In order to take the skew-symmetric information into account, the problem can be treated in an alternative way. From a geometric point of view, the solution of a binary classification problem is given by a hyperplane or some type of decision surface. If it is possible to solve a classification problem in this way, then the following topologic assumption must be true: Given a single datum, points in a sufficiently small neighborhood should belong to the same class (excluding points lying on the decision surface). As a consequence, if we are going to classify a data set relying on a given similarity matrix, points close to each other using such similarities should in general belong to the same class. Therefore, we should construct a similarity matrix $K^{*}$ such that $K^{*}\left(x_{i}, x_{j}\right)$ should be large for $x_{i}$ and $x_{j}$ in the same class, and small for $x_{i}$ and $x_{j}$ in different classes. Since we have two (possibly non-equivalent) sources of information, $s_{i j}$ and $s_{j i}$, we should define $K^{*}\left(x_{i}, x_{j}\right)$ as a function $h\left(s_{i j}, s_{j i}\right)$ that conforms to the preceding rule. We can adopt a simple and intuitive choice:

$$
K^{*}\left(x_{i}, x_{j}\right)= \begin{cases}\max \left(s_{i j}, s_{j i}\right), & \text { if } y_{i}=y_{j}, \\ \min \left(s_{i j}, s_{j i}\right), & \text { if } y_{i} \neq y_{j}\end{cases}
$$

In this way, if $x_{i}$ and $x_{j}$ are in the same class, it is guaranteed that $K^{*}\left(x_{i}, x_{j}\right)$ will be the largest possible, according to the available information. If $x_{i}$ and $x_{j}$ belong to different classes, we can expect a low similarity between them, and this is achieved by the choice $K^{*}\left(x_{i}, x_{j}\right)=\min \left(s_{i j}, s_{j i}\right)$. Hence, the method tends to move closer those points belonging to the same class, and tends to separate points belonging to different classes. This similarity matrix $K^{*}$ is symmetric, and $K^{*}=S$ when $S$ is symmetric. Note that this similarity matrix makes sense only for classification tasks, since we need the class labels to build it.

The problem above can be formulated as the combination of two similarity matrices. Let $K_{1}$ and $K_{2}$ be two matrices defined, respectively, from the upper and lower triangular parts of $S$, that is:

$$
K_{1}\left(x_{i}, x_{j}\right)=\left\{\begin{array}{ll}
s_{i j}, & \text { if } i \leq j, \\
s_{j i}, & \text { if } i>j,
\end{array} \quad K_{2}\left(x_{i}, x_{j}\right)= \begin{cases}s_{j i}, & \text { if } i \leq j, \\
s_{i j}, & \text { if } i>j .\end{cases}\right.
$$

Note that $K_{1}$ and $K_{2}$ have the same diagonal elements. Equation (6) can be stated as follows:

$$
K^{*}\left(x_{i}, x_{j}\right)= \begin{cases}\max \left(K_{1}\left(x_{i}, x_{j}\right), K_{2}\left(x_{i}, x_{j}\right)\right), & \text { if } y_{i}=y_{j}, \\ \min \left(K_{1}\left(x_{i}, x_{j}\right), K_{2}\left(x_{i}, x_{j}\right)\right), & \text { otherwise. }\end{cases}
$$

The problem of classifying data with the asymmetric similarity matrix $S$ is thus translated to the problem of combining the two symmetric similarity matrices $K_{1}$ and $K_{2}$, giving rise to an output symmetric similarity matrix $K^{*}$. This scheme can be expressed in a matrix-like form. Let $y$ denote the vector of labels, where for simplicity $y_{i} \in\{-1,+1\}$ (the extension to the multilabel case is straightforward). Consider the matrix $Y=\operatorname{diag}(y)$, whose diagonal entries are the $y_{i}$ labels. It is immediate to show that (7) is equivalent to:

$$
K^{*}\left(x_{i}, x_{j}\right)=\frac{1}{2}\left(K_{1}\left(x_{i}, x_{j}\right)+K_{2}\left(x_{i}, x_{j}\right)\right)+\frac{1}{2} y_{i} y_{j}\left|K_{1}\left(x_{i}, x_{j}\right)-K_{2}\left(x_{i}, x_{j}\right)\right|,
$$

and (7) reduces to:

$$
K^{*}=\frac{1}{2}\left(K_{1}+K_{2}\right)+\frac{1}{2} Y\left|K_{1}-K_{2}\right| Y .
$$

Note the similarity between this expression and the decomposition of an asymmetric matrix shown in (5). In addition to the skew-symmetric information, (9) takes into account the 
label information. Sections 3 and 4 will be devoted to extend the previous motivation into two general methodologies.

\section{Quantifying the difference of information: the general case}

In this section we will extend the motivation in Sect. 3 to a more general methodology for the construction of a single similarity matrix from a collection of similarity matrices. As already mentioned, we will focus on Step 2 of Algorithm 1. By analogy with the reasonings in Sect. 3, we will derive matrix combinations of the form:

$$
K^{*}=\frac{1}{2}\left(K_{1}+K_{2}\right)+\tau Y g\left(K_{1}-K_{2}\right) Y,
$$

where $g\left(K_{1}-K_{2}\right)$ is a function that quantifies the difference of information between matrices $K_{1}$ and $K_{2}$, and $\tau$ is a positive constant used to control the relative importance of this difference. If $K_{1}$ and $K_{2}$ tend to produce the same classification results, then $g\left(K_{1}-K_{2}\right)$ should become almost null, so that (10) would yield $K^{*} \simeq K_{1} \simeq K_{2}$. This will be the usual scheme that we will follow. Moreover, the methods will be generalized for the combination of more than two similarity matrices. In the following, we will refer to the input matrices of the training algorithm as kernel matrices. This makes sense since our classification setting is that of Support Vector methods and, as described in Sect. 2, building a similarity matrix from a kernel matrix is straightforward. In fact, a kernel matrix often behaves like a similarity matrix. For instance, this is the case for matrices built using the RBF kernel $K_{c}\left(x_{i}, x_{j}\right)=e^{-\left\|x_{i}-x_{j}\right\|^{2} / c}$.

Let $K_{1}, K_{2}, \ldots, K_{M}$ be the set of $M$ input kernel matrices available (defined on the data set $\left\{x_{1}, \ldots, x_{l}\right\}$ ), and denote by $K^{*}$ the desired output combination. The extension of (10) to the combination of more than two kernel matrices is straightforward:

$$
K^{*}=\bar{K}+\tau Y \sum_{i<j} g\left(K_{i}-K_{j}\right) Y,
$$

where $\bar{K}$ is the average of the kernel matrices and $g$, as before, quantifies the difference of information among the kernel matrices involved. Expression (11) can be generalized as:

$$
\bar{K}+\tau Y g\left(\operatorname{Var}\left(K_{1}, K_{2}, \ldots, K_{M}\right)\right) Y,
$$

where $\operatorname{Var}\left(K_{1}, K_{2}, \ldots, K_{M}\right)$ is a measure of the variability within the kernel matrices. To alleviate the notation, in the following let $V=\sum_{i<j} g\left(K_{i}-K_{j}\right)$, so that (11) can be restated as:

$$
K^{*}=\bar{K}+\tau Y V Y .
$$

Within this framework, several choices for $V$ will be developed in the rest of this section. Notice that, since $K^{*}$ is not necessarily a linear combination of kernel matrices, positive definiteness of $K^{*}$ is not guaranteed and the comments in Sect. 2.3 apply.

\subsection{Average kernel method}

In the simplest case, if we take $V=C$, where $C$ is a matrix of constants $c_{i j}$, we obtain the component-wise expression $K^{*}\left(x_{i}, x_{j}\right)=\bar{K}\left(x_{i}, x_{j}\right)+\tau c_{i j} y_{i} y_{j}$. Notice that the labels 
and the constants are needed to evaluate the kernel, which is a restriction at testing time. Within our framework, Algorithm 2 makes use of conditional labels at testing time, but the constants have to be determined. A possibility is to make $c_{i j}=0$, leading to $K^{*}=\bar{K}$. This is equivalent to not taking into account the difference of information among the matrices. The component-wise expression for $K^{*}$ is then: $K^{*}\left(x_{i}, x_{j}\right)=\bar{K}\left(x_{i}, x_{j}\right)$. In this way, the present point of view provides a new interpretation for a long familiar and intuitive method. This method will be referred in the following as AKM ('Average Kernel Method').

\subsection{The Absolute Value (AV) method}

Next we propose a method that generalizes (10) for the combination of more than two kernel matrices. To measure the variability within the kernel matrices we will follow the $L_{1}$ approach by considering $V=\sum_{m=1}^{M}\left|K_{m}-\bar{K}\right|$ in (13). Thus, the desired output $K^{*}$ is built using the component-wise formula:

$$
K^{*}\left(x_{i}, x_{j}\right)=\bar{K}\left(x_{i}, x_{j}\right)+\tau y_{i} y_{j} \sum_{m=1}^{M}\left|K_{m}\left(x_{i}, x_{j}\right)-\bar{K}\left(x_{i}, x_{j}\right)\right|,
$$

where $\tau$ is a positive constant to control the relative importance given to the difference of information among kernel matrices. Equation (14) constitutes the true generalization of (9). In the following, this method will be referred as AV ('Absolute Value' method).

\subsection{The 'Pick-out' method}

Next we show how to generalize (7) for the combination of more than two kernel matrices. Although this method does not fit in the general scheme of (10), it is interesting to remark that it suits with the particular case $M=2$ and $\tau=\frac{1}{2}$. The generalization is straightforward by considering:

$$
K^{*}\left(x_{i}, x_{j}\right)= \begin{cases}\max _{1 \leq m \leq M} K_{m}\left(x_{i}, x_{j}\right), & \text { if } y_{i}=y_{j}, \\ \min _{1 \leq m \leq M} K_{m}\left(x_{i}, x_{j}\right), & \text { if } y_{i} \neq y_{j}\end{cases}
$$

In this way, if $x_{i}$ and $x_{j}$ are in the same class, it is guaranteed that $K^{*}\left(x_{i}, x_{j}\right)$ will be the largest possible according to the available information. If $x_{i}$ and $x_{j}$ belong to different classes, we can expect a low similarity between them, and this is achieved by the choice of the minimum kernel value. In the following this method will be referred as the 'Pick-out' method. Formulas (7)-(9) show that the AV method reduces to the Pick-out method when two kernel matrices are being combined and $\tau=\frac{1}{2}$ in (14). As already mentioned, this is not true for the general case $M>2$.

\subsection{Generalization properties}

In order to measure the generalization properties of our output kernel matrices, we will use the measure introduced in Cristianini et al. (2002). Given two kernel matrices $K_{1}$ and $K_{2}$, their empirical alignment is defined as:

$$
A\left(K_{1}, K_{2}\right)=\frac{\left\langle K_{1}, K_{2}\right\rangle}{\sqrt{\left\langle K_{1}, K_{1}\right\rangle\left\langle K_{2}, K_{2}\right\rangle}},
$$


where $\left\langle K_{1}, K_{2}\right\rangle=\sum_{i, j}^{l} K_{1}\left(x_{i}, x_{j}\right) K_{2}\left(x_{i}, x_{j}\right)$ denotes the Frobenius inner product between matrices. The empirical alignment $A\left(K_{1}, K_{2}\right)$ is a similarity measure between kernel matrices. It can be shown that if there is a high alignment between a given kernel $K$ and the ideal kernel $K_{o}=y * y^{T}$, where $y$ is the column vector of labels, then the kernel $K$ will have good theoretical generalization properties (Cristianini et al. 2002). Although these measures are interesting from a theoretical point of view, they might have limited practical relevance. Therefore, in the experimental section we will provide extensive numerical results that support the effectiveness of our proposals.

In the next proposition we show that the AV method improves the AKM method. The proposition provides a lower bound for the $\tau$ value which will be used in the experiments to fix the final value of $\tau$.

Proposition 1 The empirical alignment of the AV kernel matrix with the ideal kernel is higher than the empirical alignment of the AKM kernel matrix with the ideal kernel if $\tau$ is such that $\tau\left[S(V)^{2}-l^{2} S(V \otimes V)\right] \geq 2 l^{2}\langle\bar{K}, Y V Y\rangle-2 S(V)\left\langle\bar{K}, K_{o}\right\rangle$ and $\tau \geq$ $\max \left(-\frac{\left\langle\bar{K}, K_{o}\right\rangle}{S(V)}, 0\right)$, where $S(V)=\sum_{i, j} V\left(x_{i}, x_{j}\right)$ and ' $\otimes$ ' denotes the element by element product between matrices (Hadamard product).

Proof See Appendix B.

Regarding the Pick-out method, Proposition 1 applies for the case $\tau=1 / 2$ and $M=2$.

\section{Weighting methods}

In this section, we will propose several new combination methods based on the concept of functional combination of kernel matrices, which extends the concept of linear combination of kernel matrices. To motivate the approach, consider the situation in Fig. 1. It is a twoclass problem where the data in each class are grouped in two clusters. In this situation, the starting point are two linear kernel functions, $K_{1}$ and $K_{2}$, from which the linear discrimination functions $f_{1}(x)$ and $f_{2}(x)$ have been calculated. We seek the best linear combination $K=\lambda_{1} K_{1}+\lambda_{2} K_{2}$. Notice that this combination scheme fits into the well known mixture models of distributions (McLachlan and Peel 2000) or, in the context of Neural Networks the mixture of experts (ME) model (Jacobs et al. 1991). The induced SVM classifier will take the form:

$$
f(x)=\sum_{i} \alpha_{i} K\left(x, x_{i}\right)=\lambda_{1} \sum_{i} \alpha_{i} K_{1}\left(x, x_{i}\right)+\lambda_{2} \sum_{i} \alpha_{i} K_{2}\left(x, x_{i}\right),
$$

thus, also a linear classifier (a straight line since $K_{1}$ and $K_{2}$ are linear kernels). Hence, it is clear that no constants $\lambda_{1}$ and $\lambda_{2}$ exist to solve the classification problem. However, if the $\lambda_{i}$ are functions of the form $\lambda_{i}(x, z)$, the idealistic solution would be simply to take $\lambda_{1}(x, z)=1, \lambda_{2}(x, z)=0$ for data points in the $A$ and $B$ clouds on the left hand side of the figure, and $\lambda_{2}(x, z)=1, \lambda_{1}(x, z)=0$ for data points in the $A$ and $B$ clouds on the right hand side of the figure.

In the general case, for the set of kernel matrices $K_{1}, K_{2}, \ldots, K_{M}$, consider the following (functional) weighted sum:

$$
K^{*}=\sum_{m=1}^{M} W_{m} \otimes K_{m},
$$


Fig. 1 Example of non-separable groups using linear combinations of kernels

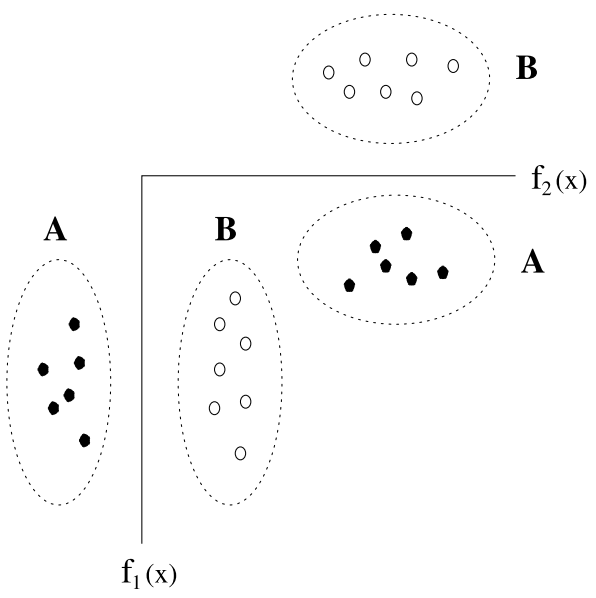

where $W_{m}=\left[w_{m}\left(x_{i}, x_{j}\right)\right]$ is a matrix whose elements are nonlinear functions $w_{m}\left(x_{i}, x_{j}\right)$, with $x_{i}$ and $x_{j}$ data points in the sample. We assume that $K_{m}\left(x_{i}, x_{j}\right) \in[0,1] \forall i, j, m$ (otherwise they can be scaled). Notice that if $w_{m}\left(x_{i}, x_{j}\right)=\mu_{m}$, where $\mu_{m}, m=1, \ldots, M$ are constants, then the method reduces to calculate a simple linear combination of matrices:

$$
K^{*}=\sum_{m=1}^{M} \mu_{m} K_{m}
$$

As mentioned in Sect. 1, in Lanckriet et al. (2004) a method is suggested to learn the coefficients $\mu_{m}$ of the linear combination by solving a semi-definite programming problem. So, the formulation used in Lanckriet et al. (2004) is a particular case of the formula we use. If we take $\mu_{m}=\frac{1}{M}$, the average of the kernel matrices (the AKM method described in Sect. 4.1) is obtained.

Regarding our proposals, consider the $(i, j)$ element of the matrix $K^{*}$ in (17):

$$
K^{*}\left(x_{i}, x_{j}\right)=\sum_{m=1}^{M} w_{m}\left(x_{i}, x_{j}\right) K_{m}\left(x_{i}, x_{j}\right) .
$$

This is the general formula of the approach that we will follow. Given that $K^{*}$ is not necessarily a linear combination of kernel matrices, positive definiteness of $K^{*}$ is not guaranteed and the comments in the previous sections apply.

Next we will show how to calculate the weighting functions $w_{m}\left(x_{i}, x_{j}\right)$. To this aim, we will make use of conditional class probabilities. Consider the pair $\left(x_{i}, y_{i}\right)$ and an unlabeled observation $x_{j}$. Given the observed value $x_{j}$, define $P\left(y_{i} \mid x_{j}\right)$ as the probability of $x_{j}$ being in class $y_{i}$. If $x_{i}$ and $x_{j}$ belong to the same class this probability should be high. Unfortunately, this probability is unknown and has to be estimated. In our proposals, we will estimate it by $P\left(y_{i} \mid x_{j}\right)=\frac{n_{i j}}{n}$, where $n_{i j}$ is the number of the $n$-nearest neighbors of $x_{j}$ belonging to class $y_{i}$, determined in the feature space induced by each kernel.

Notice that each kernel induces a different type of neighborhood. Hence, it is advisable to estimate this probability for each kernel representation, that is, for the kernel $K_{m}$ we will estimate the conditional probabilities $P_{m}\left(y_{i} \mid x_{j}\right)$.

To estimate the conditional class probabilities, the appropriate size of the neighborhood could be determined by cross-validation or using the optimal value $k=l^{\frac{4}{d+4}}$ (see 
Fig. 2 MDS for a single kernel

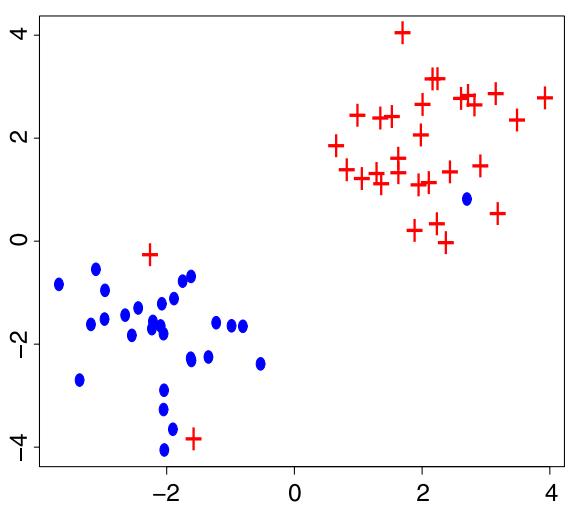

Silverman 1986), where $l$ is the number of observations, and $d$ is the dimension of the problem (this is the typical approach for the $k$-nearest neighbor classifier). In case of no vectorial objects available, only the cross-validation option is valid. We propose a dynamic alternative method: given two points $x_{i}$ and $x_{j}$, we look for the first common neighbor. To be more specific, let $R\left(x_{i}, n\right)=\left\{n\right.$-nearest neighbors of $\left.x_{i}\right\}$, and choose $k=\operatorname{argmin}_{n}\left\{R\left(x_{i}, n\right) \cap R\left(x_{j}, n\right) \neq \emptyset\right\}$. In this way, for each data point $\left(x_{i}\right.$ and $\left.x_{j}\right)$, the size $k$ of the neighborhood is determined by the number of neighbors nearer than the first common neighbor.

Obviously, the size $k$ of the neighborhood depends on the particular pair of points under consideration. Note that this is a method free of parameters: there is no need to fix the an a priori size of the neighborhood.

\subsection{The exponential weighting scheme ('ExpWS')}

To motivate our first weighting method, consider the example in Fig. 2. It represents the first two coordinates obtained using multidimensional scaling for one single kernel over a training data set. The weight $w_{m}\left(x_{i}, x_{j}\right)$ that should be assigned to this kernel in (19) depends on the pair of points $\left(x_{i}, x_{j}\right)$ we are taking into account. For most pairs $\left(x_{i}, x_{j}\right)$ the kernel suits well, but there are three points clearly surrounded by points in the other class. Six possible situations are represented in Figs. 3(a) to 3(f):

(a) Two points, both in different classes, but surrounded by points in their own class. In this case the kernel is working properly. The two points under consideration belong to different classes and the similarity between them, respect to all the other similarities, is such that they are clearly in different areas of the space. We are interested in a method that assigns a high value to $w_{m}\left(x_{i}, x_{j}\right)$.

(b) Two points, both in different classes, and surrounded by points in the other class. In this case the kernel is clearly not working because $x_{i}$ and $x_{j}$ belong to different classes and they lie in the wrong area of the space. The neighbors of point $x_{i}$ belong to the class of point $x_{j}$ and the neighbors of point $x_{j}$ belong to the class of point $x_{i}$. We are interested in a method that assigns a low value to $w_{m}\left(x_{i}, x_{j}\right)$.

(c) Two points, both in the same class, and surrounded by points in their own class. The kernel is working right for this pair of points, therefore, we are interested in a method that, in this situation, assigns a high value to $w_{m}\left(x_{i}, x_{j}\right)$. 


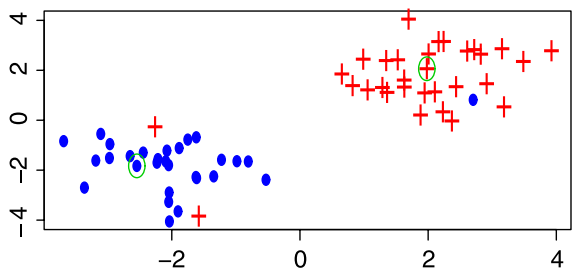

a

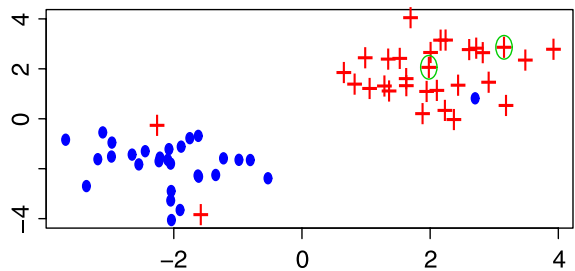

C

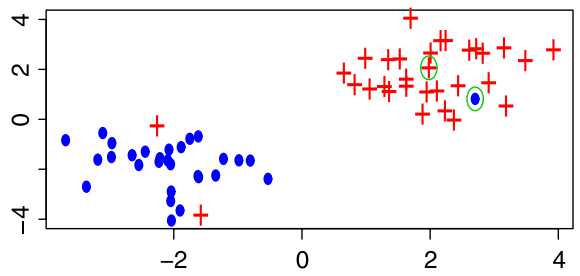

e

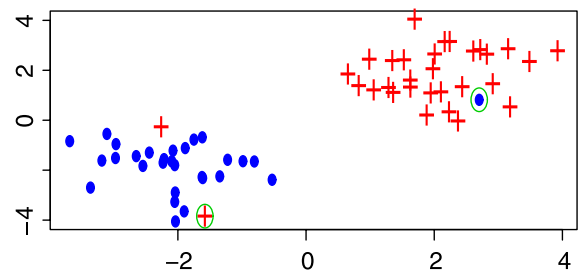

b

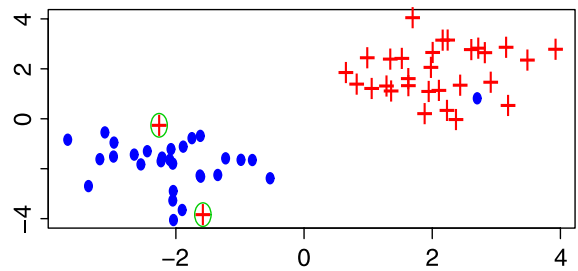

d

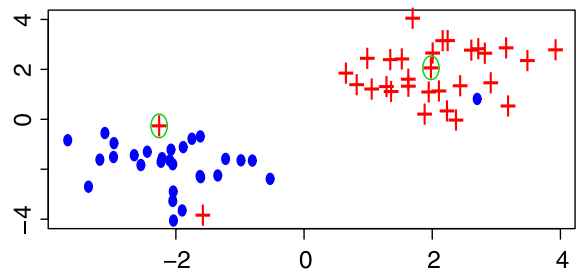

f

Fig. 3 Possible situations: the considered pair of points within each case is marked

(d) Two points, both in the same class, but surrounded by points in the other class. The two points under consideration belong to the same class, but their neighborhoods belong to the other class. We are interested in a method that assigns a low value to $w_{m}\left(x_{i}, x_{j}\right)$.

(e) Two points, both in different classes, one of them surrounded by points in its own class and the other surrounded by points in the other class. We are interested in a method that assigns a low value to $w_{m}\left(x_{i}, x_{j}\right)$.

(f) Two points, both in the same class, one of them surrounded by points in their own class and the other surrounded by points in the other class. We are interested in a method that assigns a low value to $w_{m}\left(x_{i}, x_{j}\right)$.

We have to define a method that integrates all the available information, namely: the kernel $K_{m}\left(x_{i}, x_{j}\right)$, the neighborhood of the points $x_{i}$ and $x_{j}$, and the label information.

The method we propose is influenced by the ideas in Muñoz and Villagarcía (1998) and in Domeniconi et al. (2000), where the variables are weighted according to their relative discrimination power. We make use of similar ideas to raise the weight of kernels with expected good classification performance and, analogously, to diminish the influence of less informative kernels. 
Let

$$
\rho_{m}\left(x_{i}, x_{j}\right)=\frac{P_{m}\left(y_{i} \mid x_{j}\right)+P_{m}\left(y_{j} \mid x_{i}\right)}{2}=\frac{n_{i j}+n_{j i}}{2 n} .
$$

Let

$$
\bar{P}\left(y_{i} \mid x_{j}\right)=\frac{1}{M} \sum_{m=1}^{M} P_{m}\left(y_{i} \mid x_{j}\right), \quad \bar{\rho}\left(x_{i}, x_{j}\right)=\frac{\bar{P}\left(y_{i} \mid x_{j}\right)+\bar{P}\left(y_{j} \mid x_{i}\right)}{2},
$$

and

$$
r_{m}\left(x_{i}, x_{j}\right)=\frac{\left(\bar{\rho}\left(x_{i}, x_{j}\right)-\rho_{m}\left(x_{i}, x_{j}\right)\right)^{2}}{\rho_{m}\left(x_{i}, x_{j}\right)} .
$$

The value of $r_{m}\left(x_{i}, x_{j}\right)$ will be inversely related to the relative difference between $K_{m}$ and the average of the whole set of kernel matrices. That is, the numerator in (21) approaches zero when the information conveyed by $K_{m}$ tends to be similar to the information collected by the entire set of kernel matrices. Now, we construct $w_{m}\left(x_{i}, x_{j}\right)$ as a function of $r_{m}\left(x_{i}, x_{j}\right)$. The relative relevance of kernel $K_{m}$ can be evaluated by:

$$
w_{m}\left(x_{i}, x_{j}\right)=\gamma\left(x_{i}, x_{j}\right) \mathrm{e}^{\tau / r_{m}\left(x_{i}, x_{j}\right)} .
$$

We call this method 'Exponential Weighting Scheme' (ExpWS). The parameter $\gamma\left(x_{i}, x_{j}\right)$ assures that the sum of the weights over the kernels is 1 for the pair $\left(x_{i}, x_{j}\right)$. The parameter $\tau$ is used to control the influence of $r_{m}\left(x_{i}, x_{j}\right)$ on $w_{m}\left(x_{i}, x_{j}\right)$. If $\tau=0$, this influence is ignored, and the method reduces to the AKM method. On the other hand, for large values of $\tau$, changes in $r_{m}$ will be exponentially reflected in $w_{m}$. The weighting scheme defined by (22) increases the weight of $K_{m}\left(x_{i}, x_{j}\right)$ if $\rho_{m}\left(x_{i}, x_{j}\right)$ is high and $x_{i}$ and $x_{j}$ belong to the same class (Fig. 3(c)), or if $\rho_{m}\left(x_{i}, x_{j}\right)$ is low and $x_{i}$ and $x_{j}$ belong to different classes (Fig. 3(a)). On the other hand, the weight $w_{m}\left(x_{i}, x_{j}\right)$ will be low if the points under consideration belong to the same class but $\rho_{m}\left(x_{i}, x_{j}\right)$ is low (Fig. 3(d)), or if they belong to different classes but $\rho_{m}\left(x_{i}, x_{j}\right)$ is high (Fig. 3(b)). Notice that, Figs. 3(e)-3(f) show non-extreme cases and therefore the weight of $K_{m}\left(x_{i}, x_{j}\right)$ will take values neither close to 0 nor close to 1 .

\subsection{The 'MaxMin' method}

This method produces a functional combination of two kernels, namely, the maximum and the minimum of the ordered sequence of kernels, zero being the weight assigned to the rest of the kernels. Consider the ordered sequence:

$$
\min _{1 \leq m \leq M} K_{m}\left(x_{i}, x_{j}\right)=K_{[1]}\left(x_{i}, x_{j}\right) \leq K_{[2]}\left(x_{i}, x_{j}\right) \leq \cdots \leq K_{[M]}\left(x_{i}, x_{j}\right)=\max _{1 \leq m \leq M} K_{m}\left(x_{i}, x_{j}\right),
$$

where the subscript $[\cdot]$ denotes the position induced by the order. This method builds each element of $K^{*}$ using the formula:

$$
K^{*}\left(x_{i}, x_{j}\right)=\bar{\rho}\left(x_{i}, x_{j}\right) K_{[M]}\left(x_{i}, x_{j}\right)+\left(1-\bar{\rho}\left(x_{i}, x_{j}\right)\right) K_{[1]}\left(x_{i}, x_{j}\right) .
$$

If $x_{i}$ and $x_{j}$ belong to the same class then the conditional class probabilities $\bar{\rho}\left(x_{i}, x_{j}\right)$ will be high and the method guarantees that $K^{*}\left(x_{i}, x_{j}\right)$ will be large. On the other hand, if $x_{i}$ and $x_{j}$ belong to different classes the conditional class probabilities $\bar{\rho}\left(x_{i}, x_{j}\right)$ will be low and the method will produce a value close to the minimum of the kernels. In the following, this 
Table 1 Summary of the data sets used in the experiments

\begin{tabular}{lccl}
\hline Database & Number of data & Dimension & Classes \\
\hline Two Servers & 300 & 2 & 2 \\
Comp. Information & 400 & 2 & 2 \\
Comp. Attributes & 200 & 4 & 2 \\
Glass & 214 & 9 & 2 \\
Cancer & 683 & 9 & 2 \\
Handwritten Digit & 1128 & 848 & 2 \\
Shuttle & 253 & 6 & 2 \\
Ionosphere & 351 & 34 & 2 \\
Heart & 462 & 9 & 2 \\
New-Thyroid & 215 & 5 & 3 \\
Hepatitis & 155 & 19 & 2 \\
Vote & 435 & 16 & 2 \\
Mfeat & 600 & 649 & 3 \\
Term categorization & 982 & 600 & 2 \\
\hline
\end{tabular}

method will be referred as MaxMin. If we fix $\bar{\rho}\left(x_{i}, x_{j}\right)=1$ for $x_{i}$ and $x_{j}$ belonging to the same class, and $\bar{\rho}\left(x_{i}, x_{j}\right)=0$ for $x_{i}$ and $x_{j}$ belonging to different classes, then the MaxMin method (23) reduces to the Pick-out method (15). Therefore, the 'Pick-out' method is the limiting case of the 'MaxMin' method.

\section{Experiments}

To test the performance of the proposed methods, a SVM has been trained on several artificial and real data sets using the kernel matrix $K^{*}$ constructed. For the AV method (based on quantifying the difference of information among kernels), the value of $\tau$ has been chosen taking as a reference the lower bounds provided by Proposition 1. For the ExpWS, the value of the parameter $\tau$ has been assigned via cross-validation. The description of the data sets can be consulted in Table 1 .

We have compared the proposed methods with a variety of classifiers, including some of the most successful ones for the combination of matrices: Multivariate Additive Regression Splines (MARS) (Hastie et al. 2001), Logistic Regression (LR), Linear Discriminant Analysis (LDA), $k$-Nearest Neighbor classification $(k-\mathrm{NN}$, using crossvalidation to choose the optimal value $k$ around the value suggested in (see Silverman 1986) $k=l^{\frac{4}{d+4}}$, where $l$ is the number of observations, and $d$ is the dimension of the problem), a standard SVM (that we will denote by SSVM) using a RBF kernel $K_{c}\left(x_{i}, x_{j}\right)=e^{-\left\|x_{i}-x_{j}\right\|^{2} / c}$, with $c$ and the regularization parameters chosen using the crossvalidation methodology described in Keerthi and Lin (2003), and the method proposed in Lanckriet et al. (2004). For the last method, we use the implementation developed in Bousquet and Herrmann (2003).

In order to compare the performance of the methods, the average of the classification errors, standard deviations, and the sensitivity and specificity measures have been calculated over the 10 runs of each technique, that is, each data set has been randomly partitioned 10 times in a training and a testing set, and a run of the experiment has been done over each partition.

In order to compare our methods with the other combination techniques (MARK-L and SDP), we have carried out a Wilcoxon signed-rank test (see, for instance, Lehmann 1975). 
Table 2 Summary of the percentage of misclassified data for the individual kernels, the proposed methods for kernel combination and the best alternative method. The best option is presented in bold

\begin{tabular}{|c|c|c|c|c|c|c|c|}
\hline Database & $\begin{array}{l}\text { Best/worst } \\
\text { single }\end{array}$ & AV & Pick-out & ExpWs & MaxMin & \multicolumn{2}{|c|}{$\begin{array}{l}\text { Best alternative } \\
\text { method }\end{array}$} \\
\hline Two Servers & $0.0 / 50.0$ & 6.0 & 6.0 & 2.3 & 3.5 & 3.3 & SDP \\
\hline Comp. Inf. & $15.0 / 15.0$ & 4.3 & 4.3 & 3.8 & 5.0 & 3.9 & SSVM/MARS \\
\hline Comp. Var. & $24.2 / 24.6$ & 20.6 & 20.6 & 4.8 & 3.2 & 6.6 & $k-\mathrm{NN}$ \\
\hline Glass & $8.2 / 10.3$ & 7.7 & 8.6 & 7.9 & 6.4 & 8.2 & MARS \\
\hline Cancer 1 & $3.7 / 7.8$ & 3.1 & 3.2 & 2.9 & 2.9 & 2.6 & $k-\mathrm{NN}$ \\
\hline Cancer 2 & $3.1 / 24.7$ & 3.1 & 3.4 & 3.1 & 2.8 & 2.6 & $k-\mathrm{NN}$ \\
\hline Handwritten Digit & $4.7 / 12.7$ & 4.0 & 6.3 & 3.8 & 3.4 & 1.5 & $k-\mathrm{NN}$ \\
\hline Shuttle & $2.5 / 48.3$ & 3.2 & 4.5 & 4.0 & 4.0 & 4.6 & AKM \\
\hline Ionosphere & $7.1 / 37.2$ & 6.8 & 7.8 & 7.3 & 6.5 & 6.3 & SSVM \\
\hline Heart-Disease & $27.6 / 30.9$ & 26.3 & 27.0 & 31.5 & 26.1 & 26.5 & $k-\mathrm{NN}$ \\
\hline New-Thyroid & $6.0 / 20.9$ & 4.2 & 3.3 & 4.4 & 4.0 & 4.7 & MARK-L \\
\hline Hepatitis & $16.5 / 21.3$ & 16.5 & 16.7 & 17.2 & 14.3 & 17.4 & $\mathrm{AKM} / k-\mathrm{NN}$ \\
\hline Vote & $4.0 / 25.6$ & 3.4 & 4.3 & 3.1 & 3.1 & 3.7 & AKM/SSVM \\
\hline Mfeat & $1.8 / 36.7$ & 0.9 & 3.5 & 1.0 & 1.0 & 0.8 & SSVM \\
\hline Term categorization & $-1-$ & 0.8 & 1.4 & 1.4 & 1.2 & 1.4 & SDP/AKM \\
\hline
\end{tabular}

This nonparametric test is used to compare the median of the results for different runs of each method. So, the null hypothesis of the test is that our methods do not improve the existing combination techniques.

In Sect. 6.1 we present a summary of the main classification results. For the details we refer to Appendix A, where the results at training and testing time for all the proposed and alternative methods and data set are shown.

\subsection{Summary results}

Table 2 shows a summary of the main experimental results. For each data set, we present the classification results for the best and worst single kernels used in the combination. The results for the AV, Pick-out, ExpWS and MaxMin methods are also shown. In addition we show the result for the best alternative method in each experiment. The presented results correspond to the percentage of misclassified data at testing time.

The AV method obtains the best result in one out of fifteen experiments. Similarly, the Pick-out, ExpWS and MaxMin methods obtain the best results in one, three, and five out of fifteen experiments, respectively. $k$-NN, SSVM, AKM, SDP, MARS and MARK-L were the best alternative method in six, four, four, two, two and one of fifteen experiments, respectively.

The values in each entry of Table 3 refer to how many times the method in the column has been better/same/worse than the method in the row, over all the data sets.

We have sorted the methods according to their average rank, following the approach described in Demsar (2006), and Kuncheva and Rodríguez (2007). The best method has rank 1 , the second best has rank 2, and so forth. If there are ties, the average of the ranks is assigned to each method. The final rank of a method is measured by its average rank over all data sets. Table 4 shows the average ranks for the proposed and alternative methods. To do 


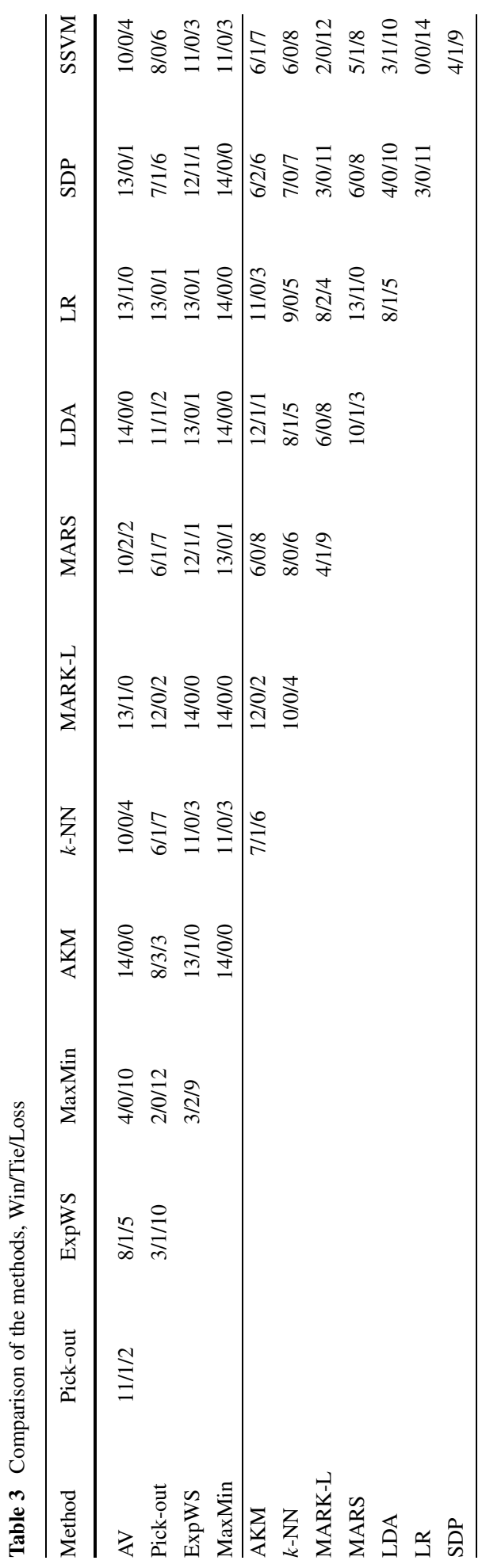


Table 4 Win-Tie-Loss and Benefit regarding the best alternative. Win, Loss and Dominance from all pairwise comparisons

\begin{tabular}{|c|c|c|c|c|c|c|}
\hline \multirow[t]{2}{*}{ Method } & \multirow[t]{2}{*}{ Rank } & \multicolumn{2}{|l|}{ Best alternative } & \multicolumn{3}{|c|}{ All pairwise } \\
\hline & & Win-Tie-Loss & Benefit & Win & Loss & Dominance \\
\hline MaxMin & 2.2 & $8-0-6$ & 10.5 & 135 & 16 & 119 \\
\hline AV & 3.3 & $7-0-7$ & 4 & 119 & 29 & 90 \\
\hline ExpWS & 3.5 & $7-1-6$ & 7 & 115 & 31 & 84 \\
\hline Pick-out & 6.0 & $3-1-10$ & -42 & 80 & 66 & 14 \\
\hline SSVM & 5.9 & $0-4-10$ & -40 & 83 & 65 & 18 \\
\hline$k-\mathrm{NN}$ & 6.6 & $0-6-8$ & -50.5 & 75 & 78 & -3 \\
\hline MARS & 6.8 & $0-2-12$ & -53 & 69 & 77 & -8 \\
\hline AKM & 7.1 & $0-4-10$ & -57.5 & 64 & 81 & -17 \\
\hline SDP & 7.4 & $0-2-12$ & -61 & 63 & 87 & -24 \\
\hline LDA & 9.4 & $0-0-14$ & -89 & 34 & 114 & -80 \\
\hline MARK-L & 9.6 & $0-1-13$ & -93 & 31 & 119 & -88 \\
\hline LR & 10.3 & $0-0-14$ & -101.5 & 22 & 127 & -105 \\
\hline
\end{tabular}

Fig. 4 Average rank and confidence interval for the proposed and alternative methods

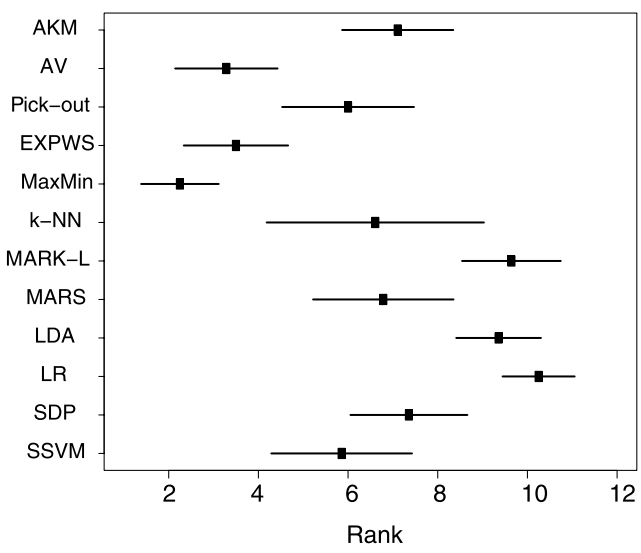

this, we define a measure, called Benefit, as the sum, over all the data sets, of the differences between the rank of a method and the rank of the best alternative. The last column, the Dominance, is defined as the difference between Wins and Losses, where Wins is the total number of times the corresponding method has improved another one, and Losses is the total number of times the method has been worse than another method.

The new proposals obtain the best results. The MaxMin method achieves the best rank (2.2). MaxMin, AV and ExpWS are the only methods with positive Benefit and Dominance. For the Pick-out method, usually a better alternative method can be found. This is the reason why the Benefit of the Pick-out method is negative. Nevertheless, the Dominance is positive given that the Pick-out method outperforms most of the alternative methods. All the alternative techniques achieve negative Benefits and only the SSVM method achieves positive dominance. Figure 4 shows the average ranks and their corresponding $95 \%$ confidence intervals for the methods. 


\section{Conclusions}

In this work we have proposed several techniques for the combination of similarity matrices within the context of SVM classifiers. The proposed framework is based on the natural idea that individuals belonging to the same class should be similar. This is supported by the fact that the suggested methods compare favorably theoretically and computationally to other well established classification techniques (and also to other techniques for the combination of kernel matrices) in a variety of artificial and real data sets.

The proposed new methods improve, in general, the performance of some of the most successful state of the art techniques. In particular, the best overall schemes are the techniques based on the combination of kernels and the use of local neighborhood information (the AV, MaxMin and ExpWS methods). Although the results are very promising there is still room for improvement. According to the numerical results, some recipes of use can be given. For high dimensional settings, the AV method seems to be the most appropriate. However, for low dimensional environments, the MaxMin and ExpWS methods show a better performance. The Pick-out method, although showing an improved performance regarding other methods in the literature, has a slightly more variable behavior. This is due to the fact that the method does not make use of local neighborhood information.

Regarding further research, a natural extension is to study the application of this methodology to non-supervised problems (see, for instance, Muñoz and Moguerza 2006), and straightforwardly its use within other kernel-based classification methods.

Acknowledgements The authors thank Prof. Robert P.W. Duin and Elżbieta Pȩkalska for useful discussions. This work was partially supported by Spanish grants MEC MTM2004-02334, MEC MTM2006-14961C05-05 and URJC-CM-2006-CET-0391.

\section{Appendix A}

\section{A.1 Artificial data sets}

\section{A.1.1 The two-servers data base}

The data set contains 300 data points in $\mathbb{R}^{2}$. There are two linearly separable groups. This data set illustrates the situation of two groups of computers (depending on two servers) sending e-mails among them. Denote by $d_{m}\left(x_{i}, x_{j}\right), m=1,2$, the time that a message takes to travel from computer $x_{i}$ to computer $x_{j}$. We have defined two kernel matrices $K_{1}$ and $K_{2}$ respectively by: $K_{m}\left(x_{i}, x_{j}\right)=1-d_{m}\left(x_{i}, x_{j}\right) / \max \left\{d_{m}\left(x_{i}, x_{j}\right)\right\}, m=1,2$, where $d_{m}\left(x_{i}, x_{j}\right)$ denotes Euclidean distances, and we have corrupted the entries of the matrices at random: for each pair $\left(x_{i}, x_{j}\right)$, one element of the pair $\left(K_{1}\left(x_{i}, x_{j}\right), K_{2}\left(x_{i}, x_{j}\right)\right)$ has been substituted by a random number in $[0,1]$. Therefore, some entries in $K_{1}$ and $K_{2}$ are randomly corrupted, but taking the correct information from each matrix the problem would be perfectly solvable. Thus, it is not possible to find a kernel $K^{*}=\lambda_{1} K_{1}+\lambda_{2} K_{2}$ that models the problem correctly. The difference between $K_{1}\left(x_{i}, x_{j}\right)$ and $K_{2}\left(x_{i}, x_{j}\right)$ is explained by the different ways in which the information may travel between $x_{i}$ and $x_{j}$. Usually, the saturation in a computer network implies that the quickest path is not the shortest path and, therefore, it is not systematically true that $d_{1}\left(x_{i}, x_{j}\right)<d_{2}\left(x_{i}, x_{j}\right)$ (or the opposite). The randomness has been introduced to simulate this phenomenon.

Since we are introducing information about labels in the proposed methods, we expect that our constructed kernel matrices will be useful for data visualization. To check this conjecture, we represent the first two coordinates obtained using MDS on the kernel matrices. 


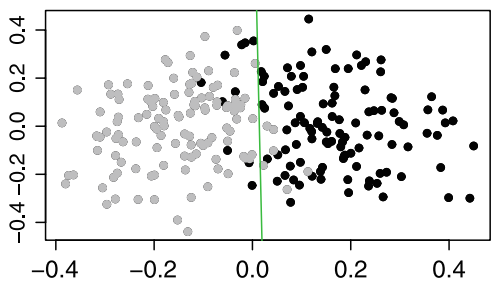

(a) MDS for AKM matrix

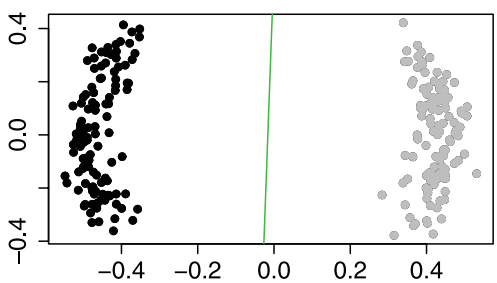

(c) MDS for Pick Out matrix

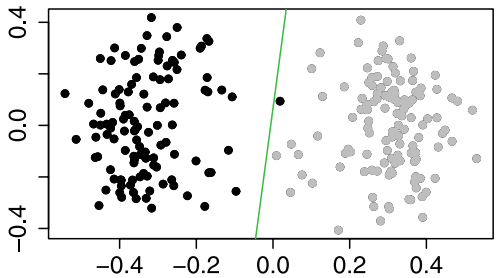

(e) MDS for MaxMin matrix

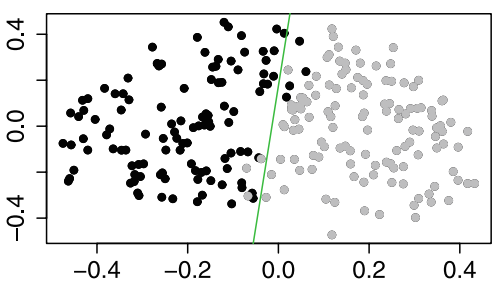

(g) MDS for SDP matrix

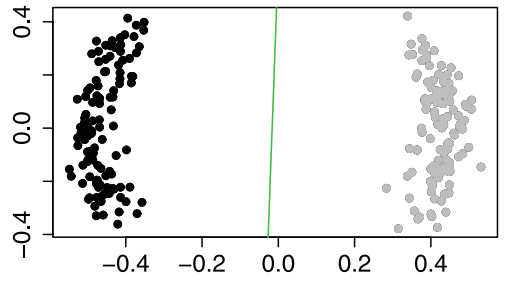

(b) MDS for AV matrix

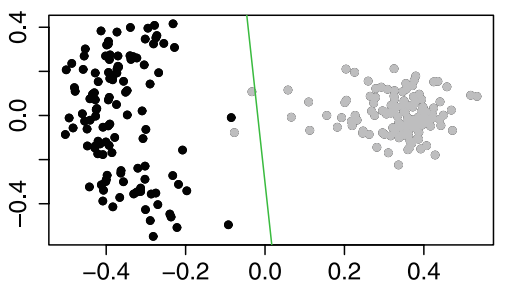

(d) MDS for ExpWS matrix

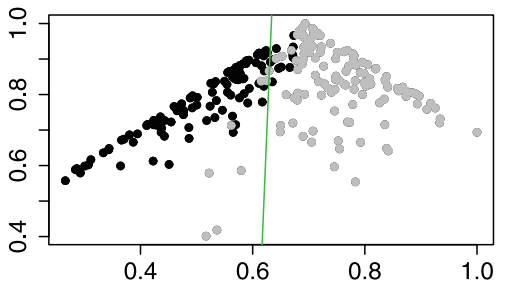

(f) MDS for MARK-L matrix

Fig. 5 Multidimensional Scaling (MDS) representation of the output kernels and SVM hyperplane for the two-servers data base for the training set (using their training labels to build the kernel matrix)

The result is shown in Fig. 5, and confirms our hypothesis. The best graphical separation between groups is achieved by the AV and the Pick-out methods. Notice that Fig. 5 shows the representation of the training data using their real labels. However, to calculate the classification error, the classification rule gives the possibility of predicting the class of the points conditioning to each class, respectively. That is the reason why the training error is larger than zero even for visually perfectly separated data. Nevertheless, it is important to remark 
Table 5 Percentage of misclassified data, sensitivity (Sens.), specificity (Spec.) and percentage of support vectors for the two-servers database. Standard deviations in brackets

\begin{tabular}{|c|c|c|c|c|c|c|c|}
\hline \multirow[t]{2}{*}{ Method } & \multicolumn{3}{|l|}{ Train } & \multicolumn{3}{|l|}{ Test } & \multirow{2}{*}{$\begin{array}{l}\text { Support } \\
\text { vectors }\end{array}$} \\
\hline & Error & Sens. & Spec. & Error & Sens. & Spec. & \\
\hline AKM & $6.8(2.0)$ & 0.966 & 0.970 & $9.7(3.6)$ & 0.896 & 0.915 & $50.2(3.5)$ \\
\hline AV & $3.1(0.7)$ & 0.966 & 0.970 & $6.0(2.9)$ & 0.933 & 0.948 & $8.2(0.4)$ \\
\hline Pick-out & $1.5(0.6)$ & 0.984 & 0.984 & $6.0(3.5)$ & 0.928 & 0.952 & $4.2(0.4)$ \\
\hline ExpWS & $2.2(1.1)$ & 0.973 & 0.979 & $2.3(2.9)$ & 0.962 & 0.992 & $19.0(1.8)$ \\
\hline MaxMin & $2.3(1.4)$ & 0.973 & 0.980 & $3.5(2.5)$ & 0.957 & 0.971 & $13.3(1.2)$ \\
\hline MARK-L & 4.0 (1.9) & 0.976 & 0.946 & $4.5(2.2)$ & 0.961 & 0.946 & $0.8(0.0)$ \\
\hline SDP & $2.6(0.9)$ & 0.982 & 0.966 & $3.3(3.0)$ & 0.958 & 0.958 & $39.9(2.9)$ \\
\hline
\end{tabular}

that perfect separation (zero error rate of misclassified training data) is not the ultimate goal, since it may mean perfect overfitting of a given data set. As we will show in our experiments, the proposed new methods provide training error rates of misclassified training data whose relation with the testing error rates is reasonable.

In order to compare the performance of the methods, the average results over 10 runs of each technique are shown in Table 5. The standard deviations have been calculated over the 10 runs (no subsampling has been done). We use MDS to solve the problem of building a positive semi-definite kernel matrix. In this case we have taken into account only the two highest eigenvalues, so we work on a two-dimensional space. The ExpWS method achieve the best performance (a test error of 2.3\%). Regarding the Wilcoxon signed-rank test for the comparison of our methods with the MARK-L technique, the $p$-values are smaller than 0.05 for ExpWS method. So the improvement obtained by the use of our proposals is statistically significant. The results of the Pick-out method improves those obtained by using the AKM method (a test error of $6.0 \%$ vs. 9.7\%), achieving the Pick-out method the smallest number of support vectors $(4.2 \%$ vs. $50.2 \%$ in AKM).

\section{A.1.2 A false two-groups classification problem}

A natural question is whether our methods will separate any data set with arbitrary labels. They should not. To test this hypothesis we have generated a normal spherical cloud in $\mathbb{R}^{2}$, and assigned random labels to the data points. In this case there is no continuous classification surface able to separate the data in two classes. As expected, the classification error rates are close to $50 \%$ for each of the proposed methods.

\section{A.1.3 Two kernels with complementary information}

This data set consists of 400 two-dimensional points (200 per class). Each group corresponds to a normal cloud with mean vector $\mu_{i}$ and symmetric covariance matrix with diagonal elements equaling 1 , and non-diagonal elements being $\sigma_{i j}=0.7$ for the first group and $\sigma_{i j}=-0.9$ for the second group. Here $\mu_{1}=(3,3), \mu_{2}=(5,5)$. We have defined two kernels from the projections of the data set onto the coordinate axes. We have used $80 \%$ of the data for training and $20 \%$ for testing. The point in this example is that, separately, both kernels achieve a poor result (a test error of 15\%).

Although out of the scope of this paper, we have used this data set to compare the different approaches to solve the problem of building a positive definite matrix. Table 6 shows the 
Table 6 Classification errors for the kernels with complementary information, using several methods to solve the problem of building a positive definite matrix (Train error \%, Test error \%, Support Vectors \%). The best solution for each method is marked in bold font

\begin{tabular}{lll}
\hline Method & MDS & $K^{* 2}$ \\
\hline AV & $\mathbf{( 4 . 4 , 4 . 3 , ~ 2 1 . 5 )}$ & $(6.4,6.5,35.4)$ \\
Pick-out & $(\mathbf{4 . 4 , 4 . 3 , 2 1 . 5 )}$ & $(5.9,6.9,3.7)$ \\
ExpWS & $(6.4,6.5,35.4)$ & $(\mathbf{3 . 6}, \mathbf{3 . 8}, \mathbf{1 6 . 7})$ \\
MaxMin & $\mathbf{( 4 . 9 , 5 . 0 , \mathbf { 2 6 . 5 } )}$ & $(4.6,5.8,9.8)$ \\
\hline
\end{tabular}

Table 7 Percentage of misclassified data, sensitivity (Sens.), specificity (Spec.) and percentage of support vectors for the kernels with complementary information. Standard deviations in brackets

\begin{tabular}{|c|c|c|c|c|c|c|c|}
\hline \multirow[t]{2}{*}{ Method } & \multicolumn{3}{|l|}{ Train } & \multicolumn{3}{|l|}{ Test } & \multirow{2}{*}{$\begin{array}{l}\text { Support } \\
\text { vectors }\end{array}$} \\
\hline & Error & Sens. & Spec. & Error & Sens. & Spec. & \\
\hline AKM & $6.4(1.6)$ & 0.872 & 1.000 & $6.5(1.8)$ & 0.868 & 1.000 & $35.4(4.0)$ \\
\hline AV & $4.4(2.4)$ & 0.940 & 0.973 & $4.3(1.4)$ & 0.935 & 0.978 & $21.5(2.4)$ \\
\hline Pick-out & $4.4(2.4)$ & 0.940 & 0.973 & $4.3(1.4)$ & 0.935 & 0.978 & $21.5(2.4)$ \\
\hline ExpWS & $3.6(1.0)$ & 0.938 & 0.991 & $3.8(1.7)$ & 0.935 & 0.988 & $16.7(4.1)$ \\
\hline MaxMin & $4.9(1.2)$ & 0.904 & 0.998 & $5.0(1.4)$ & 0.902 & 0.996 & $26.5(2.2)$ \\
\hline$k-\mathrm{NN}$ & $3.4(1.2)$ & 0.948 & 0.985 & $4.0(1.3)$ & 0.937 & 0.982 & \\
\hline MARK-L & $6.9(1.0)$ & 0.864 & 1.000 & $7.6(3.0)$ & 0.849 & 1.000 & $33.3(0.0)$ \\
\hline MARS & $3.4(1.1)$ & 0.945 & 0.987 & $3.9(1.5)$ & 0.941 & 0.980 & \\
\hline LDA & $7.5(1.5)$ & 0.851 & 1.000 & $7.8(2.6)$ & 0.842 & 1.000 & \\
\hline LR & $7.6(1.5)$ & 0.850 & 1.000 & $7.7(2.8)$ & 0.843 & 1.000 & \\
\hline SDP & $12.0(5.6)$ & 0.853 & 0.906 & $12.9(5.2)$ & 0.839 & 0.903 & $43.3(8.8)$ \\
\hline SSVM & $3.6(1.1)$ & 0.932 & 0.996 & $3.9(1.4)$ & 0.924 & 0.996 & $26.5(3.2)$ \\
\hline
\end{tabular}

results obtained with the diverse combination methods. The AKM method does not appear on this table because the output matrix obtained using this method is, in fact, a kernel matrix. Using $K^{* 2}$ as kernel involves significantly less support vectors than using the other methods. Looking at our experimental results, the use of MDS or $K^{* 2}$ seems to be a reasonable choice (see Table 6 for the details).

Table 7 shows that the best results, in general, are obtained using our combination methods. In particular, the ExpWS method attains the best overall results (test error of 3.8\%). In this case, the defined kernel matrices (being defined from projections) convey less information about the data set than the original Euclidean The $k$-NN, LR, LDA, MARS and SVM methods start from the original data points coordinates (not from the previously defined kernel matrices). This is the main reason why in this example their performance is nearly optimal. Nevertheless, most of our methods achieve very similar results to those obtained using these classical methods, and improve the alternative methods that use the same information, SDP and MARK-L (test error of $12.9 \%$ and $8.4 \%$ respectively). Regarding the Wilcoxon signed-rank test for the comparison of our methods with these two combination techniques, the $p$-values are smaller than 0.05 for all our methods. So the improvement achieved by our methods is statistically relevant. 
Table 8 Percentage of misclassified data, sensitivity (Sens.), specificity (Spec.) and percentage of support vectors for the complementary attributes information database. Standard deviations in brackets

\begin{tabular}{|c|c|c|c|c|c|c|c|}
\hline \multirow[t]{2}{*}{ Method } & \multicolumn{3}{|l|}{ Train } & \multicolumn{3}{|l|}{ Test } & \multirow{2}{*}{$\begin{array}{l}\text { Support } \\
\text { vectors }\end{array}$} \\
\hline & Error & Sens. & Spec. & Error & Sens. & Spec. & \\
\hline$K_{1}$ & $25.6(4.2)$ & 0.769 & 0.712 & $24.6(3.6)$ & 0.786 & 0.745 & $64.2(6.6)$ \\
\hline$K_{2}$ & $24.4(1.7)$ & 0.746 & 0.807 & $24.2(2.3)$ & 0.712 & 0.807 & $62.0(3.1)$ \\
\hline AKM & $19.4(1.8)$ & 0.814 & 0.789 & $21.6(4.7)$ & 0.777 & 0.799 & $58.4(2.5)$ \\
\hline AV & $19.0(3.1)$ & 0.774 & 0.836 & $21.0(3.9)$ & 0.786 & 0.936 & $38.2(4.1)$ \\
\hline Pick-out & $22.0(1.9)$ & 0.741 & 0.816 & $20.6(4.3)$ & 0.756 & 0.827 & $15.2(1.9)$ \\
\hline ExpWS & $3.6(1.5)$ & 0.955 & 0.973 & $4.8(3.6)$ & 0.890 & 0.897 & $24.8(5.0)$ \\
\hline MaxMin & $3.0(1.6)$ & 0.956 & 0.968 & $3.2(1.9)$ & 0.961 & 0.977 & $25.6(3.0)$ \\
\hline$k-\mathrm{NN}$ & $6.0(0.6)$ & 0.942 & 0.936 & $6.6(1.7)$ & 0.942 & 0.930 & \\
\hline MARK-L & $19.2(1.8)$ & 0.790 & 0.872 & $21.0(4.9)$ & 0.787 & 0.783 & \\
\hline MARS & $14.4(2.3)$ & 0.818 & 0.888 & $19.4(3.2)$ & 0.749 & 0.872 & \\
\hline LDA & $20.6(3.2)$ & 0.778 & 0.807 & $21.6(3.2)$ & 0.781 & 0.810 & \\
\hline LR & $21.2(3.4)$ & 0.765 & 0.806 & $21.0(2.3)$ & 0.758 & 0.813 & \\
\hline SDP & $20.0(1.9)$ & 0.779 & 0.815 & $19.2(3.6)$ & 0.775 & 0.845 & $60.8(2.2)$ \\
\hline SSVM & $2.2(1.9)$ & 0.974 & 0.981 & $6.8(3.6)$ & 0.894 & 0.966 & $63.8(21.6)$ \\
\hline
\end{tabular}

\section{A.1.4 Two kernels with complementary attributes}

In this example, we build a data set made up of 200 four-dimensional points (100 per class). To build the set, we first generate four bidimensional normal clouds, with mean vectors $\mu_{i}$ $\left(\mu_{1}=(2,6), \mu_{2}=(6,6), \mu_{3}=(2,2)\right.$ and $\left.\mu_{4}=(6,2)\right)$ and covariance matrix the identity matrix. Then, we build the data set as follows: attributes 1 and 2 for the first 100 observations correspond to the points on clouds 1 and 2, attributes 3 and 4 for the last 100 observations correspond to the points on clouds 3 and 4 . The matrix is completed (attributes 3 and 4 for the first 100 observations and attributes 1 and 2 for the last 100 observations) using random numbers. The labels are positive for points on clouds 1 and 4 , and negative for points on clouds 2 and 3 . In this way, we have defined two bidimensional linearly separable data sets: the first one with the data from points on clouds 1 and 2 and the second one from points on clouds 3 and 4 .

The point in this example is that we have several attributes useful to separate a subsample of the data set, but no single attribute is able to separate the complete data set.

We have combined two RBF kernels: the first one defined using the first two attributes but not the last ones, and the second one defined using the last attributes but not the first ones. We use $K^{* 2}$ to solve the problem of building a positive semi-definite kernel matrix. We have used $50 \%$ of the data for training and $50 \%$ for testing.

The results are presented in Table 8 . Notice that separately, both kernels achieve a very poor result. The ExpWS and MaxMin methods achieve the best results, outperforming not only the individual kernels, but the alternative techniques too. Only the comparison with the SSVM method implies a non-significant difference. In this case, the AV and Pick-out methods obtain similar results to the alternative techniques. Notice that, the $k-\mathrm{NN}$ and the SSVM are the best alternative methods. The excellent ExpWS and MaxMin results are achieved because, by construction, the ExpWS and MaxMin methods use the $k$-nearest neighbor information and a SVM type classifier. 
Table 9 Percentage of overlap, test errors into the overlap and the no-overlap areas, and percentage of global error for the glass data set

\begin{tabular}{lllll}
\hline Method & \% overlap & $\begin{array}{l}\text { Overlap } \\
\text { error }\end{array}$ & $\begin{array}{l}\text { No-overlap } \\
\text { error }\end{array}$ & $\begin{array}{l}\text { Global } \\
\text { error }\end{array}$ \\
\hline AKM & - & - & - & 8.79 \\
AV & 10.28 & 34.41 & 4.13 & 7.66 \\
Pick-out & 67.85 & 14.33 & 0.44 & 8.56 \\
ExpWS & 0.93 & 33.33 & 7.24 & 7.85 \\
MaxMin & 14.77 & 22.38 & 3.78 & 6.36 \\
\hline
\end{tabular}

\section{A.2 Real data sets}

\section{A.2.1 Glass identification data set}

In this section, we will work with a database from the UCI Machine Learning Repository: the Glass Identification Database (Blake and Merz 1998). The data set consists of 214 observations with 9 features each. We will use this data set to study the 'well-posedness' of Algorithm 2. As explained in Sect. 2.2, for those points $x$ such that $\operatorname{sign}\left(f_{+}(x)\right)=\operatorname{sign}\left(f_{-}(x)\right)$, the a priori assumption on the class of $x$ is not relevant for the a posteriori classification of $x$ provided by Algorithm 2. In this context, we define the overlap as the set of voting discrepancies, that is, the set of points such that the a posteriori classifications are different: $\operatorname{sign}\left(f_{+}(x)\right) \neq \operatorname{sign}\left(f_{-}(x)\right)$. As stated in Sect. 2.2, points in the overlap area should not be classified. However, Algorithm 2 forces the classification of the voting discrepancies: points in the overlap are assigned to the class corresponding to the largest distance from each conditional discrimination hyperplane. To show that forcing the classification of voting discrepancies is better that leaving them unclassified, what remains is to check if the overall errors of our methods remain lower than the standard combination techniques errors (the AKM method), and, in fact, that is what happens.

With this aim, we have combined three different kernels on the Glass data set: a polynomial kernel $K_{1}(x, z)=\left(1+x^{T} z\right)^{2}$, a RBF kernel $K_{2}(x, z)=\mathrm{e}^{-\|x-z\|^{2} / 100}$ and a linear kernel $K_{3}(x, z)=x^{T} z$. We have normalized the kernel matrices: $K(x, z)=$ $K(x, z) / \sqrt{K(x, x) * K(z, z)}$. The results, averaged over 10 runs of the experiment, are shown in Table 9.

Even when we force the classification of the voting discrepancies, the global errors for all our methods are lower than the error obtained for the AKM method.

In addition, the MaxMin method achieves the best overall performance between the proposed methods. As expected, the test error is higher in the overlap area than in the no-overlap area for all our methods. The improvement obtained by the use of our proposals is much higher in the no-overlap area. Notice that the highest percentage of overlap and the lowest error in the no-overlap area are obtained for the Pick-out method.

Table 10 presents the results obtained when several classification methods were used. Maxmin outperforms the best individual kernel, using significantly less support vectors. MARS was the best alternative method. Regarding the Wilcoxon signed-rank test for the comparison of our methods with the MARK-L and SDP techniques, again the $p$-values are smaller than 0.05 for Maxmin, AV and ExpWS methods.

\section{A.2.2 The cancer data set}

In this section we have dealt with a database from the UCI Machine Learning Repository: the Breast Cancer data set (Mangasarian and Wolberg 1990). The data set consists 
Table 10 Percentage of misclassified data, sensitivity (Sens.), specificity (Spec.) and percentage of support vectors for the Glass database. Standard deviations in brackets

\begin{tabular}{|c|c|c|c|c|c|c|c|}
\hline \multirow[t]{2}{*}{ Method } & \multicolumn{3}{|l|}{ Train } & \multicolumn{3}{|l|}{ Test } & \multirow{2}{*}{$\begin{array}{l}\text { Support } \\
\text { vectors }\end{array}$} \\
\hline & Error & Sens. & Spec. & Error & Sens. & Spec. & \\
\hline$K_{1}$ : Polynomial & $8.2(1.5)$ & 0.663 & 1.000 & $10.1(2.3)$ & 0.581 & 0.995 & $44.9(2.7)$ \\
\hline$K_{2}: \mathrm{RBF}$ & $1.1(1.5)$ & 0.979 & 0.992 & $8.2(1.7)$ & 0.766 & 0.964 & $10.5(2.8)$ \\
\hline$K_{3}$ : Linear & $0.0(0.0)$ & 1.000 & 1.000 & $10.3(2.2)$ & 0.650 & 0.971 & $16.8(2.9)$ \\
\hline $\mathrm{AKM}$ & $0.0(0.0)$ & 1.000 & 1.000 & $8.8(1.6)$ & 0.871 & 0.730 & $15.0(1.9)$ \\
\hline $\mathrm{AV}$ & $0.2(0.4)$ & 1.000 & 0.998 & $7.7(0.8)$ & 0.785 & 0.964 & $9.5(2.4)$ \\
\hline Pick-out & $3.6(11.7)$ & 0.961 & 0.967 & $8.6(3.1)$ & 0.781 & 0.957 & $6.7(2.0)$ \\
\hline ExpWS & $0.4(0.8)$ & 1.000 & 0.995 & $7.9(1.9)$ & 0.806 & 0.964 & $14.6(3.1)$ \\
\hline MaxMin & $0.9(0.9)$ & 0.970 & 0.997 & $6.4(1.5)$ & 0.825 & 0.971 & $7.7(2.0)$ \\
\hline$k-\mathrm{NN}$ & $5.8(1.7)$ & 0.814 & 0.982 & $10.7(1.7)$ & 0.637 & 0.971 & \\
\hline MARK-L & $10.7(3.7)$ & 0.615 & 0.988 & $13.6(1.9)$ & 0.444 & 0.985 & \\
\hline MARS & $2.4(1.1)$ & 0.944 & 0.985 & $8.2(1.8)$ & 0.796 & 0.957 & \\
\hline LDA & $4.9(1.5)$ & 0.824 & 0.993 & $9.7(1.4)$ & 0.670 & 0.971 & \\
\hline LR & $3.6(2.4)$ & 0.977 & 0.960 & $9.3(3.7)$ & 0.802 & 0.923 & \\
\hline SDP & $8.2(1.5)$ & 0.663 & 1.000 & $10.1(2.3)$ & 0.581 & 0.995 & $44.9(2.7)$ \\
\hline SSVM & $0.2(0.4)$ & 1.000 & 0.998 & $9.2(1.3)$ & 0.748 & 0.962 & $15.9(7.1)$ \\
\hline
\end{tabular}

of 683 observations with 9 features each. Following Lanckriet et al. (2004), for this data set we have combined three kernels: a polynomial kernel $K_{1}(x, z)=\left(1+x^{T} z\right)^{2}$, a RBF kernel $K_{2}(x, z)=\mathrm{e}^{-\|x-z\|^{2}}$ and a linear kernel $K_{3}(x, z)=x^{T} z$. We have normalized the kernel matrices so that their entries belong to the [0,1] interval: $K(x, z)=(K(x, z)-$ $\min (K)) /(\max (K)-\min (K))$. We have used $80 \%$ of the data for training and $20 \%$ for testing.

The results, averaged over 10 runs of the experiment, are shown in Table 11. All our methods improve the best individual SVM performance (achieved using a linear kernel). The $k$-NN method shows the best overall performance (an average test error of $2.6 \%$ ). Our methods improve the alternative methods that combine kernel information, SDP and MARK-L (average test error of $6.2 \%$ and $4.1 \%$ respectively). All our combination methods provide better results than the SVM with a single RBF kernel (an average test error of 3.9\%). The lowest average percentage of support vectors $(2.9 \%)$ is associated with the AV method, with an average test error of $3.1 \%$. Notice the relatively poor performance of SDP on this set (with a high number of support vectors, 65.5\%). This result is probably due to the strong non-linearity of the data (see, for instance, Moguerza et al. 2002). The original SDP version by Lanckriet et al. (2004) presents a similar test error result (5.7\%). In fact, regarding the Wilcoxon signed-rank test for the comparison of our methods with the SDP method, the $p$-values are smaller than 0.01 for all our methods. For the comparison with the MARK-L method, the $p$-values are smaller than 0.05 for the ExpWS, PolyWS and MaxMin methods, and smaller than 0.1 for the AV method. Therefore, methods not based on pure linear combinations of the kernel matrices seem to work better.

\section{A.2.3 An alternative to parameter selection}

It is well known that the choice of kernel parameters is often critical for the good performance of SVMs. Combining kernels provides a solution that minimizes the effect of a bad 
Table 11 Percentage of misclassified data, sensitivity (Sens.), specificity (Spec.) and percentage of support vectors for the cancer data. Standard deviations in brackets

\begin{tabular}{|c|c|c|c|c|c|c|c|}
\hline \multirow[t]{2}{*}{ Method } & \multicolumn{3}{|l|}{ Train } & \multicolumn{3}{|l|}{ Test } & \multirow{2}{*}{$\begin{array}{l}\text { Support } \\
\text { vectors }\end{array}$} \\
\hline & Error & Sens. & Spec. & Error & Sens. & Spec. & \\
\hline$K_{1}$ : Polynomial & $0.1(0.1)$ & 0.997 & 1.000 & $7.8(2.5)$ & 0.866 & 0.951 & $8.3(0.8)$ \\
\hline$K_{2}: \mathrm{RBF}$ & $0.0(0.0)$ & 1.000 & 1.000 & $10.8(1.7)$ & 1.000 & 0.837 & $65.6(1.0)$ \\
\hline$K_{2}$ : Linear & $2.6(0.5)$ & 0.969 & 0.977 & $3.7(1.8)$ & 0.962 & 0.963 & $7.1(0.8)$ \\
\hline $\mathrm{AKM}$ & $1.3(0.2)$ & 0.988 & 0.987 & $3.3(1.4)$ & 0.946 & 0.978 & $31.1(0.9)$ \\
\hline AV & $2.4(0.3)$ & 0.986 & 0.971 & $3.1(1.3)$ & 0.987 & 0.959 & $2.9(0.4)$ \\
\hline Pick-out & $2.4(0.3)$ & 0.992 & 0.968 & $3.2(1.3)$ & 0.989 & 0.957 & $5.9(0.3)$ \\
\hline ExpWS & $2.3(0.5)$ & 0.981 & 0.975 & $2.9(1.6)$ & 0.983 & 0.965 & $25.3(0.6)$ \\
\hline MaxMin & $0.7(0.1)$ & 1.000 & 0.989 & $2.9(1.4)$ & 0.967 & 0.973 & $25.3(0.6)$ \\
\hline$k-\mathrm{NN}$ & $2.2(0.4)$ & 0.972 & 0.981 & $2.6(1.3)$ & 0.972 & 0.976 & \\
\hline MARK-L & $1.8(0.7)$ & 0.999 & 0.972 & $4.1(1.5)$ & 0.994 & 0.942 & $9.2(0.0)$ \\
\hline MARS & $2.9(0.7)$ & 0.956 & 0.979 & $3.1(1.4)$ & 0.958 & 0.974 & \\
\hline LDA & $3.8(0.3)$ & 0.924 & 0.982 & $3.9(1.2)$ & 0.921 & 0.980 & \\
\hline LR & $13.2(0.8)$ & 0.851 & 0.877 & $13.0(3.0)$ & 0.857 & 0.876 & \\
\hline SDP & $0.0(0.0)$ & 1.000 & 1.000 & $6.2(1.6)$ & 0.943 & 0.935 & $65.5(1.9)$ \\
\hline SSVM & $1.6(1.2)$ & 0.970 & 0.983 & $3.9(1.5)$ & 0.966 & 0.961 & $22.4(10.5)$ \\
\hline
\end{tabular}

parameter choice. Next we illustrate this situation using a collection of RBF kernels on the cancer data set. Let $\left\{K_{1}, \ldots, K_{12}\right\}$ be a set of RBF kernels with parameters $c=0.1,1,10$, $20,30,40,50,60,70,80,90,100$ respectively. We use MDS to solve the problem of building a positive definite matrices. Table 12 shows the performance of the proposed methods when combining all these kernel matrices. Again, the results have been averaged over 10 runs. The MaxMin method improves the best RBF kernel under consideration (test errors of $2.8 \%$ vs. $3.1 \%$ ). This result are specially significant because this method is parameter free, and it could be used as an alternative to the RBF parameter selection. Nevertheless, in this example all the methodologies seem to work fairly well. In particular, the results provided by all the combination methods are not degraded by the inclusion of kernels with a bad generalization performance.

\section{A.2.4 A handwritten digit recognition problem}

The experiment in this section concerns a binary classification problem: the recognition of digits ' 7 ' and ' 9 ' from the Alpaydin and Kaynak database (Alpayadin and Kaynak 1998). The data set is made up by 1128 records, represented by $32 \times 32$ binary images. The data are divided in a testing (359 instances) and a training (769 instances) set. We have employed three different methods to specify features in order to describe the images. The first one is the $4 \times 4$ method: features are defined as the number of ones in each of the 64 squares of dimension $4 \times 4$. The second method was introduced by Frey and Slate (1991): 16 attributes are derived from the image, related to the horizontal/vertical position, width, height, etc. The last method under consideration was designed by Fukushima and Imagawa (1993): features are defined as a collection of 12 different representations in a $4 \times 4$ square. This is a typical example with several different sources of information and probably complementary. We have used these representations to calculate three kernels using the Euclidean distance. 
Table 12 Percentage of misclassified data, sensitivity (Sens.), specificity (Spec.) and percentage of support vectors for the cancer data using a battery of RBF kernels. Standard deviations in brackets

\begin{tabular}{|c|c|c|c|c|c|c|c|}
\hline \multirow[t]{2}{*}{ Method } & \multicolumn{3}{|l|}{ Train } & \multicolumn{3}{|l|}{ Test } & \multirow{2}{*}{$\begin{array}{l}\text { Support } \\
\text { vectors }\end{array}$} \\
\hline & Error & Sens. & Spec. & Error & Sens. & Spec. & \\
\hline Best RBF & $2.3(0.3)$ & 0.979 & 0.976 & 3.1 (1.6) & 0.976 & 0.966 & $13.6(1.3)$ \\
\hline Worst RBF & $0.0(0.0)$ & 1.000 & 1.000 & $24.7(2.3)$ & 1.000 & 0.627 & $74.0(2.4)$ \\
\hline$\overline{\mathrm{AKM}}$ & $1.6(0.3)$ & 0.988 & 0.981 & $3.4(1.5)$ & 0.978 & 0.966 & $21.7(1.2)$ \\
\hline AV & $3.1(0.3)$ & 0.974 & 0.966 & $3.1(1.7)$ & 0.983 & 0.961 & $6.5(0.6)$ \\
\hline Pick-out & $2.5(0.5)$ & 0.945 & 0.991 & $3.4(1.2)$ & 0.954 & 0.971 & $7.7(0.4)$ \\
\hline ExpWS & $3.0(0.4)$ & 0.974 & 0.968 & $3.1(1.5)$ & 0.981 & 0.963 & $10.5(0.7)$ \\
\hline MaxMin & $0.1(0.1)$ & 0.999 & 0.998 & $2.8(1.6)$ & 0.963 & 0.975 & $14.2(1.5)$ \\
\hline MARK-L & $0.0(0.0)$ & 1.000 & 1.000 & $3.6(1.2)$ & 0.980 & 0.956 & $18.3(0.0)$ \\
\hline SDP & $0.0(0.0)$ & 1.000 & 1.000 & $3.2(1.6)$ & 0.976 & 0.964 & $41.5(4.4)$ \\
\hline
\end{tabular}

When $K^{*}$ is not positive definite, the MDS method was used to build a psd matrix. The training and test data sets has been randomly partitioned 10 times, and a run of the experiment has been done over each partition.

The classification performance for all the methods is listed in Table 13. For each classical method we have chosen the individual representation that provides the best results, and then we check if the combination methods are able to achieve the same performance. In particular, we have taken the $4 \times 4$ representation to train the $k$-NN, MARS, LR and LDA methods. To train the SVM we have taken the Fukushima and Imagawa representation. The MaxMin and ExpWS methods achieve the best results among the methods based on the use of kernels (test error of $3.4 \%$ and $3.8 \%$ respectively). Furthermore, the AV, MaxMin, and ExpWS combinations improve the results obtained using the rest of the techniques except $k$-NN. The good performance of SVM with a single kernel is unsurprising, since this method is specially efficient when working with sparse data sets in high dimensional settings (Joachims 2002). Regarding the Wilcoxon signed-rank test for the comparison of our methods with the MARK-L technique, the $p$-values are smaller than 0.05 for MaxMin, AV and ExpWS methods. The $p$-value for the comparison of MaxMin method with the SDP technique is smaller than 0.10 .

\section{A.2.5 Space Shuttle Autolanding Domain data set}

In this section we will study the effect of random kernels on the classification task. We have dealt with a database from the UCI Machine Learning Repository: the Space Shuttle Autolanding Domain data set (Blake and Merz 1998). The data set consists of 253 observations with 6 features each. The data have been standardized. For this data set we have combined three standard kernels (linear, polynomial and RBF kernels), and three random kernels. We have normalized the kernel matrices: $K(x, z)=K(x, z) / \sqrt{K(x, x) * K(z, z)}$. We have used $70 \%$ of the data for training and $30 \%$ for testing. We have used MDS to solve the problem of building a positive semi-definite kernel matrix.

The results, averaged over 10 runs of the experiment, are shown in Table 14. As expected, the classification error rates for the random kernels are close to $50 \%$ for each of the proposed methods. The random kernels influence on our methods is lower than the influence on the alternative ones. AV method achieves the best results (test error of 3.2\%), outperforming all the alternative methods ( $p<0.05$, Wilcoxon rank test). The AV method is the only method 
Table 13 Percentage of misclassified data, sensitivity (Sens.), specificity (Spec.) and percentage of support vectors for the Handwritten Digit Recognition data set. Standard deviations in brackets

\begin{tabular}{|c|c|c|c|c|c|c|c|}
\hline \multirow[t]{2}{*}{ Method } & \multicolumn{3}{|l|}{ Train } & \multicolumn{3}{|l|}{ Test } & \multirow{2}{*}{$\begin{array}{l}\text { Support } \\
\text { vectors }\end{array}$} \\
\hline & Error & Sens. & Spec. & Error & Sens. & Spec. & \\
\hline $4 \times 4$ & $0.0(0.0)$ & 1.000 & 1.000 & 4.7 (1.9) & 0.908 & 1.000 & $5.8(0.2)$ \\
\hline Frey-Slate & $10.2(1.3)$ & 0.951 & 0.843 & $12.7(2.8)$ & 0.832 & 0.914 & $29.5(2.0)$ \\
\hline Fukushima & $0.0(0.0)$ & 1.000 & 1.000 & $4.9(1.2)$ & 0.905 & 0.998 & $11.5(1.1)$ \\
\hline AKM & $0.0(0.0)$ & 1.000 & 1.000 & $5.1(1.3)$ & 0.901 & 0.998 & $9.0(1.3)$ \\
\hline AV & $0.0(0.0)$ & 1.000 & 1.000 & $4.0(1.0)$ & 0.965 & 0.955 & $74.8(1.3)$ \\
\hline Pick-out & $2.7(1.3)$ & 0.964 & 0.964 & $6.3(2.9)$ & 0.881 & 0.995 & $2.0(0.6)$ \\
\hline ExpWS & $0.1(0.1)$ & 1.000 & 0.999 & $3.8(1.5)$ & 0.932 & 0.993 & $6.7(1.8)$ \\
\hline MaxMin & $0.0(0.0)$ & 1.000 & 1.000 & $3.4(1.4)$ & 0.936 & 0.998 & $6.5(0.9)$ \\
\hline$k-\mathrm{NN}$ & $1.0(0.6)$ & 0.995 & 0.985 & $1.5(0.9)$ & 0.974 & 0.998 & \\
\hline MARK-L & $0.0(0.0)$ & 1.000 & 1.000 & $5.7(1.3)$ & 0.890 & 0.998 & \\
\hline MARS & $0.1(0.1)$ & 0.999 & 0.999 & $4.2(1.4)$ & 0.919 & 0.998 & \\
\hline LDA & $0.2(0.3)$ & 0.998 & 0.998 & $5.3(1.4)$ & 0.899 & 0.998 & \\
\hline LR & $0.0(0.0)$ & 1.000 & 1.000 & $5.0(2.3)$ & 0.912 & 0.989 & \\
\hline SDP & $0.0(0.0)$ & 1.000 & 1.000 & 4.7 (1.4) & 0.907 & 1.000 & $9.1(1.3)$ \\
\hline SSVM & $0.0(0.0)$ & 1.000 & 1.000 & $4.7(1.0)$ & 0.910 & 0.998 & $2.2(0.5)$ \\
\hline
\end{tabular}

that improves at least one of the good kernels. ExpWS and MaxMin methods improves the SDP and MARK-L methods.

\section{A.2.6 Johns Hopkins University Ionosphere database}

In this section we have dealt with a database from the UCI Machine Learning Repository: the Johns Hopkins University Ionosphere database (Blake and Merz 1998). The data set consists of 351 observations with 34 continuous predictor attributes variables each. The data have been standardized. We have used $60 \%$ of the data for training and $40 \%$ for testing. The targets were free electrons in the ionosphere. "Good" radar returns (positive examples) are those showing evidence of some type of structure in the ionosphere. "Bad" returns (negative examples) are those that do not; their signals pass through the ionosphere. The signals are preprocessed by using the time of a pulse and the pulse number. There are 17 pulse numbers. Each case in the data set is described by two attributes per pulse number. Therefore, each element in the data set is described by 34 variables. The positive class consists of 225 examples and the negative class consists of 126 examples.

For this data set we have combined seven RBF kernels $K(x, z)=e^{-\|x-z\|^{2} /\left(2 * \sigma^{2}\right)}$ with $\sigma=1,5,10,25,20,50,100$. We have used a linear kernel $K(x, z)=x^{T} z$, and a polynomial kernel $K(x, z)=\left(1+x^{T} z\right)^{2}$ as well. We have considered normalization: $K(x, z)=$ $K(x, z) / \sqrt{K(x, x) * K(z, z)}$ to make comparable the different kernels values. We have used $K^{* 2}$ to solve the problem of building a positive semi-definite kernel matrix. We have used $60 \%$ of the data for training and $40 \%$ for testing.

The classification results are shown in Table 15. The AV, MaxMin and SSVM methods achieve the best results. Regarding the Wilcoxon signed-rank test for the comparison of 
Table 14 Percentage of misclassified data, sensitivity (Sens.), specificity (Spec.) and percentage of support vectors for the Space Shuttle data set. Standard deviations in brackets

\begin{tabular}{|c|c|c|c|c|c|c|c|}
\hline \multirow[t]{2}{*}{ Method } & \multicolumn{3}{|l|}{ Train } & \multicolumn{3}{|l|}{ Test } & \multirow{2}{*}{$\begin{array}{l}\text { Support } \\
\text { vectors }\end{array}$} \\
\hline & Error & Sens. & Spec. & Error & Sens. & Spec. & \\
\hline \multicolumn{8}{|l|}{ Standard kernels } \\
\hline$K_{1}$ : Linear & $1.7(0.7)$ & 0.978 & 0.988 & $2.5(1.9)$ & 0.974 & 0.975 & $14.4(1.6)$ \\
\hline$K_{2}$ : Polynomial & $1.0(0.7)$ & 0.993 & 0.986 & $3.0(2.3)$ & 0.980 & 0.957 & $6.9(1.6)$ \\
\hline$K_{3}: \mathrm{RBF}$ & $0.0(0.0)$ & 1.000 & 1.000 & $3.4(1.7)$ & 0.964 & 0.971 & $11.5(0.9)$ \\
\hline \multicolumn{8}{|l|}{ Random kernels } \\
\hline$K_{4}$ : Linear & $41.6(2.4)$ & 0.973 & 0.060 & $44.6(5.0)$ & 0.949 & 0.046 & $95.6(5.8)$ \\
\hline$K_{5}$ : Polynomial & $41.7(2.8)$ & 0.969 & 0.064 & $44.6(5.7)$ & 0.939 & 0.043 & $95.2(6.4)$ \\
\hline$K_{6}: \mathrm{RBF}$ & $31.6(2.0)$ & 0.845 & 0.461 & $48.3(4.5)$ & 0.696 & 0.285 & $79.5(2.7)$ \\
\hline AKM & $0.0(0.0)$ & 1.000 & 1.000 & $4.6(1.6)$ & 0.963 & 0.941 & $30.6(1.4)$ \\
\hline AV & $0.0(0.1)$ & 1.000 & 0.999 & $3.2(2.0)$ & 0.974 & 0.962 & $22.4(1.8)$ \\
\hline Pick-out & $1.9(1.3)$ & 0.977 & 0.984 & $4.5(3.0)$ & 0.949 & 0.970 & $21.5(1.6)$ \\
\hline ExpWS & $0.3(0.3)$ & 0.995 & 1.000 & $4.0(2.6)$ & 0.953 & 0.968 & $19.3(2.0)$ \\
\hline MaxMin & $0.0(0.0)$ & 1.000 & 1.000 & $4.0(1.8)$ & 0.971 & 0.946 & $18.9(2.3)$ \\
\hline$k-\mathrm{NN}$ & $6.2(1.4)$ & 0.937 & 0.939 & $8.7(3.8)$ & 0.931 & 0.894 & \\
\hline MARK-L & $0.0(0.0)$ & 1.000 & 1.000 & $9.3(3.8)$ & 0.917 & 0.898 & \\
\hline MARS & $6.7(1.3)$ & 0.885 & 1.000 & $6.5(2.9)$ & 0.887 & 0.998 & \\
\hline LDA & $6.9(1.2)$ & 0.881 & 1.000 & $6.4(2.9)$ & 0.887 & 1.000 & \\
\hline LR & $5.3(1.3)$ & 0.908 & 0.999 & $7.3(2.3)$ & 0.874 & 0.998 & \\
\hline SDP & $0.0(0.0)$ & 1.000 & 1.000 & $5.8(2.4)$ & 0.950 & 0.932 & $18.6(1.5)$ \\
\hline SSVM & $0.0(0.0)$ & 1.000 & 1.000 & $5.1(2.4)$ & 0.952 & 0.946 & $53.7(1.7)$ \\
\hline
\end{tabular}

MaxMin, AV and ExpWS method with the AKM, MARK-L, MARS, LR, LDA, and $k$-NN techniques, the $p$-values are smaller than 0.05 .

\section{A.2.7 Heart-Disease Standford database}

In this section we have deal with the Heart-Disease Standford database: a retrospective sample of males in a heart-disease high-risk region of the Western Cape, South Africa. The database were obtained from www-stat.stanford.edu/ElemStatLearn. The data set consists of 462 observations with 9 features each. The data have been standardized. For this data set we have combined five kernels: three polynomial kernels $K_{m}(x, z)=\left(1+x^{T} z\right)^{m}$ (for $m=1,2$, and 3 respectively), and two RBF kernels $K_{m}(x, z)=\mathrm{e}^{-\|x-z\|^{2} / c_{m}}\left(c_{4}=50\right.$ and $\left.c_{5}=200\right)$. We have normalized the kernel matrices: $K(x, z)=K(x, z) / \sqrt{K(x, x) * K(z, z)}$. We have used $K^{* 2}$ to solve the problem of building a positive semi-definite kernel matrix. We have used $80 \%$ of the data for training and $20 \%$ for testing.

The results, averaged over 10 runs of the experiment, are shown in Table 16 . The best classification results were obtained for the MaxMin, AV and $k$-NN methods. These methods were statistically better ( $p<0.05$ for the Wilcoxon rank test) than the AKM, and MARK-L methods. In addition, The Pick-out method outperforms the AKM and MARK-L methods. The percentage of support vectors used by the Pick-out, MaxMin and AV methods were consistently lower than the percentage of support vectors used by the kernel methods under consideration (individual kernels, AKM, SDP and SSVM). 
Table 15 Percentage of misclassified data, sensitivity (Sens.), specificity (Spec.) and percentage of support vectors for the Ionosphere database. Standard deviations in brackets

\begin{tabular}{|c|c|c|c|c|c|c|c|}
\hline \multirow[t]{2}{*}{ Method } & \multicolumn{3}{|l|}{ Train } & \multicolumn{3}{|l|}{ Test } & \multirow{2}{*}{$\begin{array}{l}\text { Support } \\
\text { vectors }\end{array}$} \\
\hline & Error & Sens. & Spec. & Error & Sens. & Spec. & \\
\hline Best RBF & $3.6(0.7)$ & 0.986 & 0.923 & $7.1(1.2)$ & 0.985 & 0.834 & $40.8(2.2)$ \\
\hline Worst RBF & $35.0(2.0)$ & 1.000 & 0.000 & $37.2(3.0)$ & 1.000 & 0.000 & $71.5(4.0)$ \\
\hline Linear & $4.3(0.4)$ & 0.977 & 0.920 & 12.7 (1.9) & 0.959 & 0.728 & $25.0(1.4)$ \\
\hline Polynomial & $0.0(0.0)$ & 1.000 & 1.000 & $14.6(3.6)$ & 0.955 & 0.683 & $27.3(2.0)$ \\
\hline AKM & $2.6(1.0)$ & 0.991 & 0.944 & $7.8(1.6)$ & 0.982 & 0.821 & $43.1(2.3)$ \\
\hline AV & $2.1(0.5)$ & 0.991 & 0.958 & $6.8(1.1)$ & 0.974 & 0.860 & $42.2(2.8)$ \\
\hline Pick-out & $0.9(0.4)$ & 0.997 & 0.980 & $7.8(1.5)$ & 0.944 & 0.844 & 70.7 (3.7) \\
\hline ExpWS & $3.3(1.0)$ & 0.985 & 0.934 & $7.3(1.5)$ & 0.974 & 0.847 & $24.8(2.0)$ \\
\hline MaxMin & $2.3(0.3)$ & 0.983 & 0.965 & $6.5(1.2)$ & 0.965 & 0.886 & $18.8(1.8)$ \\
\hline$k-\mathrm{NN}$ & $9.5(1.1)$ & 0.987 & 0.753 & $16.1(1.7)$ & 0.984 & 0.592 & \\
\hline MARK-L & $0.0(0.0)$ & 1.000 & 1.000 & $7.9(1.9)$ & 0.983 & 0.817 & \\
\hline MARS & $1.7(1.2)$ & 0.998 & 0.957 & $12.3(2.9)$ & 0.957 & 0.743 & \\
\hline LDA & $7.7(1.5)$ & 0.978 & 0.820 & $14.1(2.3)$ & 0.979 & 0.657 & \\
\hline LR & 2.5 (1.9) & 0.984 & 0.959 & $14.3(1.7)$ & 0.930 & 0.727 & \\
\hline SDP & $5.4(0.6)$ & 0.978 & 0.887 & $7.5(1.7)$ & 0.991 & 0.815 & $61.0(2.1)$ \\
\hline SSVM & $2.7(2.1)$ & 0.989 & 0.941 & $6.5(0.9)$ & 0.976 & 0.873 & $50.7(10.0)$ \\
\hline
\end{tabular}

\section{A.2.8 Thyroid gland data}

In this section we consider the Thyroid gland data obtained from the UCI Machine Learning Repository (Blake and Merz 1998). Five laboratory tests are used to try to predict whether a patient's thyroid belongs to one of three classes (euthyroidism, hypothyroidism or hyperthyroidism). The data set consists of 210 observations with 5 features each. The data have been standardized. We have combined four kernels: two RBF kernels $K_{1}$ and $K_{2}$ with constant $c$ taking the values 0.02 and 0.5 , respectively, a linear kernel $K_{3}(x, z)=x^{T} z$ and a polynomial kernel $K_{1}(x, z)=\left(1+x^{T} z\right)^{2}$. We have normalized the kernel matrices: $K(x, z)=K(x, z) / \sqrt{K(x, x) * K(z, z)}$. We have used $K^{* 2}$ to solve the problem of building a positive semi-definite kernel matrix. We have taken $60 \%$ of the data for training and $40 \%$ for testing.

Our methods for kernel combination achieve the best classification results. Regarding Wilcoxon signed-rank test for the comparison of the Pick-out methods with the alternative techniques, the $p$-values are smaller than 0.05 for all the comparisons. In addition, the Pickout and the AV results are obtained by using significantly less support vectors than the other techniques.

\section{A.2.9 Hepatitis domain}

In this section we have dealt with a database from the UCI Machine Learning Repository: the Hepatitis Domain data set (Blake and Merz 1998). The data set consists of 155 observations with 20 attributes each. The data have been standardized. We have combined three kernels: a polynomial kernel $K_{1}(x, z)=\left(1+x^{T} z\right)^{2}$, a RBF kernel $K_{2}(x, z)=\mathrm{e}^{-\|x-z\|^{2} /\left(2 * \sigma^{2}\right)}(\sigma=20)$, and a linear kernel $K_{3}(x, z)=x^{T} z$. We have normalized the kernel matrices: $K(x, z)=$ 
Table 16 Percentage of misclassified data, sensitivity (Sens.), specificity (Spec.) and percentage of support vectors for the Heart-Disease database. Standard deviations in brackets

\begin{tabular}{|c|c|c|c|c|c|c|c|}
\hline \multirow[t]{2}{*}{ Method } & \multicolumn{3}{|l|}{ Train } & \multicolumn{3}{|l|}{ Test } & \multirow{2}{*}{$\begin{array}{l}\text { Support } \\
\text { vectors }\end{array}$} \\
\hline & Error & Sens. & Spec. & Error & Sens. & Spec. & \\
\hline$K_{1}:$ Linear & $15.2(1.0)$ & 0.664 & 0.945 & $30.9(3.4)$ & 0.363 & 0.861 & $62.3(1.2)$ \\
\hline$K_{2}$ : Polynomial $_{2}$ & $0.0(0.0)$ & 1.000 & 1.000 & $27.6(4.5)$ & 0.473 & 0.856 & $93.4(1.6)$ \\
\hline$K_{3}$ : Polynomial $_{3}$ & $25.5(0.5)$ & 0.551 & 0.848 & $29.8(4.6)$ & 0.479 & 0.819 & $60.5(1.9)$ \\
\hline$K_{4}: \mathrm{RBF}_{50}$ & $20.8(1.2)$ & 0.585 & 0.903 & $27.6(1.6)$ & 0.419 & 0.881 & $57.8(2.0)$ \\
\hline$K_{5}: \mathrm{RBF}_{200}$ & $19.5(0.9)$ & 0.498 & 0.968 & $27.8(4.1)$ & 0.338 & 0.921 & $63.5(1.3)$ \\
\hline $\mathrm{AKM}$ & $6.6(1.4)$ & 0.897 & 0.953 & $34.8(3.3)$ & 0.511 & 0.727 & $55.5(1.8)$ \\
\hline $\mathrm{AV}$ & $25.2(1.9)$ & 0.474 & 0.895 & $26.3(1.8)$ & 0.443 & 0.887 & $0.8(0.0)$ \\
\hline Pick-out & $26.8(1.3)$ & 0.459 & 0.878 & $27.0(5.8)$ & 0.481 & 0.858 & $1.8(0.1)$ \\
\hline ExpWS & $25.9(3.6)$ & 0.665 & 0.781 & $31.5(5.1)$ & 0.507 & 0.776 & $36.9(2.5)$ \\
\hline MaxMin & $16.1(0.6)$ & 0.659 & 0.935 & $26.1(1.5)$ & 0.526 & 0.853 & $2.0(0.2)$ \\
\hline$k-\mathrm{NN}$ & $27.8(1.0)$ & 0.327 & 0.932 & $26.5(3.3)$ & 0.346 & 0.938 & \\
\hline MARK-L & $12.1(1.3)$ & 0.785 & 0.926 & $36.1(4.8)$ & 0.488 & 0.741 & \\
\hline MARS & $24.8(1.2)$ & 0.541 & 0.864 & $26.7(3.2)$ & 0.493 & 0.859 & \\
\hline LDA & $25.2(1.6)$ & 0.541 & 0.858 & $28.7(4.2)$ & 0.486 & 0.832 & \\
\hline LR & $30.2(0.9)$ & 0.807 & 0.640 & $29.8(3.7)$ & 0.796 & 0.654 & \\
\hline SDP & $17.7(0.7)$ & 0.675 & 0.901 & $30.0(5.7)$ & 0.462 & 0.825 & $57.3(1.2)$ \\
\hline SSVM & $21.6(2.6)$ & 0.585 & 0.895 & $29.1(2.7)$ & 0.364 & 0.59 & $62.1(2.8)$ \\
\hline
\end{tabular}

Table 17 Classification errors for the Thyroid gland data set

\begin{tabular}{lllr}
\hline Method & Train error $\%$ & Test error $\%$ & Support vector \% \\
\hline$K_{1}: \mathrm{RBF}_{0.02}$ & $0.0(0.0)$ & $20.9(2.2)$ & $100.0(0.0)$ \\
$K_{2}: \mathrm{RBF}_{0.5}$ & $0.0(0.0)$ & $12.3(2.9)$ & $51.8(2.3)$ \\
$K_{3}:$ Linear & $0.9(0.6)$ & $5.8(3.0)$ & $8.3(1.3)$ \\
$K_{4}:$ Polynomial & $0.0(0.0)$ & $6.0(2.1)$ & $9.6(1.2)$ \\
\hline AKM & $0.0(0.0)$ & $5.8(2.8)$ & $28.1(1.3)$ \\
AV & $2.0(0.7)$ & $4.2(2.3)$ & $3.9(0.5)$ \\
Pick-out & $1.2(0.6)$ & $3.3(1.0)$ & $3.6(0.2)$ \\
ExpWS & $0.0(0.0)$ & $4.4(1.9)$ & $10.0(1.2)$ \\
MaxMin & $0.3(0.4)$ & $4.0(1.6)$ & $5.0(0.7)$ \\
$k$-NN & $4.8(0.6)$ & $9.1(2.1)$ & \\
MARK-L & $0.0(0.0)$ & $4.7(2.2)$ & \\
MARS & $2.2(0.7)$ & $5.3(1.8)$ & \\
LDA & $2.9(0.3)$ & $6.7(2.1)$ & \\
LR & $2.6(1.0)$ & $6.7(1.5)$ & \\
SDP & $0.0(0.0)$ & $5.1(1.8)$ & $8.1(0.7)$ \\
SSVM & $1.4(0.3)$ & $5.8(2.5)$ & $26.9(1.4)$ \\
\hline
\end{tabular}

$K(x, z) / \sqrt{K(x, x) * K(z, z)}$. We use $K^{* 2}$ to solve the problem of building a positive semidefinite kernel matrix. We have used $70 \%$ of the data for training and $30 \%$ for testing. 
Table 18 Percentage of misclassified data, sensitivity (Sens.), specificity (Spec.) and percentage of support vectors for the Hepatitis database. Standard deviations in brackets

\begin{tabular}{|c|c|c|c|c|c|c|c|}
\hline \multirow[t]{2}{*}{ Method } & \multicolumn{3}{|l|}{ Train } & \multicolumn{3}{|l|}{ Test } & \multirow{2}{*}{$\begin{array}{l}\text { Support } \\
\text { vectors }\end{array}$} \\
\hline & Error & Sens. & Spec. & Error & Sens. & Spec. & \\
\hline$K_{1}$ : Polynomial & $0.0(0.0)$ & 1.000 & 1.000 & $16.5(3.7)$ & 0.451 & 0.934 & $45.3(2.8)$ \\
\hline$K_{2}: \mathrm{RBF}$ & $0.0(0.0)$ & 1.000 & 1.000 & $18.9(3.1)$ & 0.522 & 0.893 & $40.9(3.8)$ \\
\hline$K_{3}$ : Linear & $5.7(2.7)$ & 0.835 & 0.970 & $21.3(6.5)$ & 0.405 & 0.876 & $27.2(4.4)$ \\
\hline $\mathrm{AKM}$ & $0.0(0.0)$ & 1.000 & 1.000 & $17.4(3.7)$ & 0.439 & 0.922 & $43.0(3.6)$ \\
\hline AV & $4.6(1.1)$ & 0.928 & 0.961 & $16.5(5.2)$ & 0.537 & 0.905 & $16.6(1.5)$ \\
\hline Pick-out & $8.3(1.7)$ & 0.915 & 0.918 & $16.7(6.3)$ & 0.564 & 0.889 & $13.6(1.3)$ \\
\hline ExpWS & $0.7(0.9)$ & 0.985 & 0.994 & $17.2(4.4)$ & 0.543 & 0.901 & $31.9(2.4)$ \\
\hline MaxMin & $0.4(0.5)$ & 1.000 & 0.995 & $14.3(3.4)$ & 0.617 & 0.914 & $35.0(2.7)$ \\
\hline$k-\mathrm{NN}$ & $12.8(2.1)$ & 0.542 & 0.965 & $17.4(2.9)$ & 0.344 & 0.939 & \\
\hline MARK-L & $10.3(1.4)$ & 0.606 & 0.971 & $18.7(4.1)$ & 0.348 & 0.930 & \\
\hline MARS & $11.7(2.0)$ & 0.562 & 0.967 & $20.4(4.6)$ & 0.289 & 0.923 & \\
\hline LDA & $9.2(1.4)$ & 0.720 & 0.957 & $17.6(3.3)$ & 0.464 & 0.912 & \\
\hline LR & $17.1(4.3)$ & 0.902 & 0.811 & $30.9(6.9)$ & 0.775 & 0.653 & \\
\hline SDP & $0.0(0.0)$ & 1.000 & 1.000 & $18.5(4.3)$ & 0.535 & 0.890 & $38.8(3.9)$ \\
\hline SSVM & $5.1(5.1)$ & 0.747 & 0.991 & $18.0(3.2)$ & 0.554 & 0.924 & 53.5 (1.9) \\
\hline
\end{tabular}

Classification results are presented in Table 18. MaxMin, AV, and Pick-out achieve the best results. In fact, regarding the Wilcoxon signed-rank test, only the MaxMin method was significantly better than the alternative techniques ( $p$-values are smaller than 0.05 ). The remaining new proposals exhibit a similar performance to that of the best alternatives.

\section{A.2.10 1984 United States Congressional Voting Records Database}

In this section we have dealt with a database from the UCI Machine Learning Repository: the Voting Records Database (Blake and Merz 1998). The data set consists of 435 observations and 16 attributes each. The data set includes votes for each of the U.S. House of Representatives Congressmen on the 16 key votes identified by the CQA. For this data set we have combined a collection of RBF and polynomial kernels: $K_{m}(x, z)=\left(1+x^{T} z\right)^{m}(m=1,2)$ and $K_{m}(x, z)=\mathrm{e}^{-\|x-z\|^{2} / c}$, with $\sigma_{m}=0.02,2,50,200$, and 5000 (for $m=3,4,5,6$, and 7 respectively). We have considered the: $K(x, z)=K(x, z) / \sqrt{K(x, x) * K(z, z)}$ to make comparable the different kernels values. We have used $K^{* 2}$ to solve the problem of building a positive semi-definite kernel matrix. We have taken $70 \%$ of the data for training and $30 \%$ for testing.

The classification results, averaged over 10 runs of the experiment, are shown in Table 19. The ExpWS, MaxMin and AV methods achieve the best results, improving the classification results of all the individual kernels under consideration and the alternative methods (AKM, SSVM and SDP methods). Regarding the Wilcoxon rank test, the MaxMin and ExpWS methods were significantly better than the MARK-L, $k$-NN and LR method.

\section{A.2.11 Multiple features data set (Handwritten Numerals)}

This data set consists of features of handwritten numerals ('0'-'9') extracted from a collection of Dutch utility maps (Blake and Merz 1998). We will refer to this data set as Mfeat. The 
Table 19 Percentage of misclassified data, sensitivity (Sens.), specificity (Spec.) and percentage of support vectors for the Voting Records database. Standard deviations in brackets

\begin{tabular}{|c|c|c|c|c|c|c|c|}
\hline \multirow[t]{2}{*}{ Method } & \multicolumn{3}{|l|}{ Train } & \multicolumn{3}{|l|}{ Test } & \multirow{2}{*}{$\begin{array}{l}\text { Support } \\
\text { vectors }\end{array}$} \\
\hline & Error & Sens. & Spec. & Error & Sens. & Spec. & \\
\hline$K_{1}$ : Linear & $0.0(0.0)$ & 1.000 & 1.000 & $25.6(1.9)$ & 1.000 & 0.366 & $95.9(1.1)$ \\
\hline$K_{2}:$ Polynomial & $0.0(0.0)$ & 1.000 & 1.000 & $13.9(2.8)$ & 0.992 & 0.667 & $85.1(1.0)$ \\
\hline$K_{3}: \mathrm{RBF}_{0.02}$ & $1.4(0.4)$ & 0.986 & 0.986 & $4.0(0.8)$ & 0.959 & 0.962 & $85.1(1.0)$ \\
\hline$K_{4}: \mathrm{RBF}_{2}$ & $3.4(0.8)$ & 0.964 & 0.969 & $4.1(1.6)$ & 0.951 & 0.970 & $16.4(0.8)$ \\
\hline$K_{5}: \mathrm{RBF}_{50}$ & $6.0(0.8)$ & 0.934 & 0.950 & $6.3(1.5)$ & 0.938 & 0.935 & $40.3(0.9)$ \\
\hline$K_{6}: \mathrm{RBF}_{200}$ & $2.0(0.5)$ & 0.983 & 0.974 & $5.3(1.6)$ & 0.951 & 0.940 & $9.0(0.8)$ \\
\hline$K_{7}: \mathrm{RBF}_{5000}$ & $0.0(0.0)$ & 1.000 & 1.000 & $6.3(2.7)$ & 0.951 & 0.916 & $19.9(0.8)$ \\
\hline AKM & $0.0(0.0)$ & 1.000 & 1.000 & $3.7(1.0)$ & 0.962 & 0.966 & $25.7(0.7)$ \\
\hline AV & $2.1(0.5)$ & 0.980 & 0.977 & $3.4(1.6)$ & 0.969 & 0.962 & $5.5(0.7)$ \\
\hline Pick-out & $2.2(0.5)$ & 0.983 & 0.970 & $4.3(2.4)$ & 0.962 & 0.951 & $13.8(1.0)$ \\
\hline ExpWS & $0.1(0.1)$ & 1.000 & 0.998 & $3.1(1.1)$ & 0.974 & 0.962 & $21.1(0.6)$ \\
\hline MaxMin & $0.0(0.0)$ & 1.000 & 1.000 & $3.1(0.9)$ & 0.972 & 0.966 & $13.2(0.4)$ \\
\hline$k-\mathrm{NN}$ & $5.1(0.5)$ & 0.940 & 0.964 & $6.7(1.5)$ & 0.928 & 0.940 & \\
\hline MARK-L & $0.0(0.0)$ & 1.000 & 1.000 & $4.7(0.6)$ & 0.933 & 0.981 & \\
\hline MARS & $4.3(0.6)$ & 0.948 & 0.970 & $4.3(1.2)$ & 0.946 & 0.973 & \\
\hline LDA & $4.4(0.5)$ & 0.948 & 0.969 & $4.3(1.2)$ & 0.946 & 0.973 & \\
\hline LR & $2.7(0.8)$ & 0.974 & 0.972 & $5.2(1.7)$ & 0.944 & 0.955 & \\
\hline SDP & $1.7(0.3)$ & 0.981 & 0.986 & $3.8(0.9)$ & 0.961 & 0.962 & $12.2(0.4)$ \\
\hline SSVM & $1.5(1.4)$ & 0.984 & 0.986 & $3.7(1.1)$ & 0.964 & 0.963 & $24.6(9.4)$ \\
\hline
\end{tabular}

Table 20 Feature sets defined on the Mfeat handwritten numerals data set

\begin{tabular}{ll}
\hline 1. & 76 Fourier coefficients of the character shapes \\
2. & 216 profile correlations \\
3. & 64 Karhunen-Loève coefficients \\
4. & 240 pixel averages in $2 \times 3$ windows \\
5. & 47 Zernike moments \\
6. & 6 morphological features \\
\hline
\end{tabular}

experiment in this section concerns a multiple class classification problem: the recognition of digits ' 1 ', ' 7 ' and ' 9 '. 200 patterns per class (for a total of 600 patterns) have been digitized in binary images. These digits are represented in terms of the six feature sets presented in Table 20.

A different kernel have been defined using the Euclidean distance for each representation. The kernels were normalized: $K(x, z)=K(x, z) / \sqrt{K(x, x) * K(z, z)}$. We have used MDS to solve the problem of building a positive semi-definite kernel matrix. We have used $50 \%$ of the data for training and $50 \%$ for testing.

The classification performance for all the methods, averaged over 10 runs of the experiment, is listed in Table 21. For each classical method we have chosen the individual representation that provides the best results, and then we check if the combination methods are able to achieve the same performance. The SSVM, AV, ExpWS and MaxMin methods achieve the best results among the methods based on the use of kernels. These techniques improve all the individual kernels under consideration. 
Table 21 Classification errors for the Mfeat digit data set

\begin{tabular}{lccr}
\hline Method & Train error $\%$ & Test error $\%$ & Support vector \% \\
\hline$K_{1}:$ Fourier & $0.2(0.3)$ & $8.70(2.06)$ & $17.43(1.04)$ \\
$K_{2}:$ profile corr. & $0.0(0.0)$ & $2.73(1.39)$ & $6.70(0.59)$ \\
$K_{3}:$ Karhunen-Love & $0.0(0.0)$ & $2.07(0.81)$ & $10.13(0.43)$ \\
$K_{4}: 2 \times 3$ windows & $0.0(0.0)$ & $1.77(0.85)$ & $12.92(0.79)$ \\
$K_{5}:$ Zernike & $0.0(0.0)$ & $3.80(0.74)$ & $6.87(0.43)$ \\
$K_{6}:$ morphological feat. & $20.3(0.7)$ & $36.73(2.90)$ & $54.72(13.3)$ \\
\hline AKM & $0.0(0.0)$ & $1.10(0.54)$ & $11.42(0.53)$ \\
AV & $0.0(0.0)$ & $0.87(0.47)$ & $9.73(2.68)$ \\
Pick-out & $0.0(0.0)$ & $3.56(1.26)$ & $7.02(0.62)$ \\
ExpWS & $0.0(0.0)$ & $0.97(0.54)$ & $12.05(3.23)$ \\
MaxMin & $0.0(0.0)$ & $1.00(0.57)$ & $6.40(0.59)$ \\
$k$-NN & $1.40(0.52)$ & $2.50(0.76)$ & $11.75(1.41)$ \\
MARK-L & $0.0(0.0)$ & $1.77(0.47)$ & $75.57(1.51)$ \\
MARS & $0.0(0.0)$ & $4.33(0.80)$ & $10.32(2.70)$ \\
LDA & $0.0(0.0)$ & $8.70(1.60)$ & $1.23(0.50)$ \\
LR & $0.0(0.0)$ & $0.80(0.59)$ & \\
SDP & $0.0(0.0)$ & & \\
SSVM & $0.0(0.0)$ & &
\end{tabular}

\section{A.2.12 Term categorization}

The interesting task of term categorization has been studied by different authors in the last few years (see, for instance, Muñoz 1997). Its motivation is the automatic expansion of domain-specific lexicons. To check the methods in a high dimensional setting, we will work on a small text data base with two groups of documents. The first class is made up of 296 records from the LISA data base (Library Science Abstracts), with the common topic 'library science'. The second class contains 394 records on 'pattern recognition' from the INSPEC data base (bibliographic references for physics, computing and engineering research, from the IEE Institute). There is a mild overlap between the two classes, due to records dealing with 'automatic abstracting'. We select terms that occur in at least 10 documents (obtaining 982 terms). Labels are assigned to terms by voting on the classes of documents in which these terms appear. The task is to correctly predict the class of each term. Following Muñoz (1997), we have defined the kernel matrix $K_{1}$ by $K_{1}\left(x_{i}, x_{j}\right)=\frac{\left|x_{i} \wedge x_{j}\right|}{\left|x_{i}\right|}=\frac{\sum_{k}\left|\min \left(x_{i k}, x_{j k}\right)\right|}{\sum_{k}\left|x_{i k}\right|}$, where $\left|x_{i}\right|$ measures the number of documents indexed by term $x_{i}$, and $\left|x_{i} \wedge x_{j}\right|$ the number of documents indexed by both terms $x_{i}$ and $x_{j}$. Similarly, $K_{2}=\frac{\left|x_{i} \wedge x_{j}\right|}{\left|x_{j}\right|}$. Therefore, $K_{1}\left(x_{i}, x_{j}\right)$ may be interpreted as the degree in which the topic represented by term $x_{i}$ is a subset of the topic represented by term $x_{j}$. This numeric measure of subsethood is due to Kosko (1991). The task is to classify the database terms using the information provided by both kernels. Note that we are dealing with about 1000 points in 600 dimensions, and this is a near empty set. This means that it will be very easy to find a hyperplane that divides the two classes. Nevertheless, the example is still useful to illustrate the relative performance of the proposed methods. Following the scheme of the preceding examples, Table 22 shows the results. In this case, we have used $K^{* 2}$ as kernel to solve the problem of building a positive definite matrix. 
Table 22 Percentage of misclassified data, sensitivity (Sens.), specificity (Spec.) and percentage of support vectors for the term categorization example. Standard deviations in brackets. '-' indicates non convergence of the method

\begin{tabular}{|c|c|c|c|c|c|c|c|}
\hline \multirow[t]{2}{*}{ Method } & \multicolumn{3}{|l|}{ Train } & \multicolumn{3}{|l|}{ Test } & \multirow{2}{*}{$\begin{array}{l}\text { Support } \\
\text { vectors }\end{array}$} \\
\hline & Error & Sens. & Spec. & Error & Sens. & Spec. & \\
\hline AKM & $0.0(0.0)$ & 1.000 & 1.000 & $1.4(1.3)$ & 0.993 & 0.965 & $13.4(0.4)$ \\
\hline AV & $0.0(0.0)$ & 1.000 & 1.000 & $0.8(0.5)$ & 0.996 & 0.980 & $8.0(0.4)$ \\
\hline Pick-out & $0.1(0.2)$ & 1.000 & 0.998 & $1.4(0.8)$ & 0.993 & 0.966 & $6.0(0.4)$ \\
\hline ExpWS & $0.0(0.0)$ & 1.000 & 1.000 & $1.4(1.3)$ & 0.993 & 0.965 & $13.4(0.4)$ \\
\hline MaxMin & $0.0(0.0)$ & 1.000 & 1.000 & $1.2(0.8)$ & 0.996 & 0.965 & $6.9(0.6)$ \\
\hline$k-\mathrm{NN}$ & $12.8(4.1)$ & 0.997 & 0.528 & $14.0(2.4)$ & 0.990 & 0.495 & \\
\hline MARK-L & - & - & - & - & - & - & \\
\hline MARS & - & - & - & - & - & - & \\
\hline LDA & $0.0(0.0)$ & 1.000 & 1.000 & $31.4(5.8)$ & 0.720 & 0.591 & \\
\hline LR & - & - & - & - & - & - & \\
\hline SDP & $0.0(0.0)$ & 1.000 & 1.000 & $1.4(1.3)$ & 0.993 & 0.965 & $13.4(0.4)$ \\
\hline SSVM & $0.0(0.0)$ & 1.000 & 1.000 & $13.3(3.3)$ & 1.000 & 0.447 & $86.8(1.0)$ \\
\hline
\end{tabular}

Two of our four new proposals clearly outperforms the rest of the methods. In particular, the AV method achieves the best performance (test error of $0.8 \%$ vs $1.4 \%$ of SDP). The other two proposals provide similar results to the best alternatives. Notice that SVM and $k$-NN clearly underfit the data. These two methods are based on the use of a single distance (kernel in the case of SVM).

\section{Appendix B}

Proof of Proposition 1 The empirical alignments to compare are:

$$
A\left(\bar{K}, K_{o}\right)=\frac{\left\langle\bar{K}, K_{o}\right\rangle}{\sqrt{\left\langle K_{o}, K_{o}\right\rangle} \sqrt{\langle\bar{K}, \bar{K}\rangle}}=\frac{\left\langle\bar{K}, K_{o}\right\rangle}{l \sqrt{\langle\bar{K}, \bar{K}\rangle}},
$$

and

$$
A\left(K^{*}, K_{o}\right)=\frac{\left\langle K^{*}, K_{o}\right\rangle}{\sqrt{\left\langle K_{o}, K_{o}\right\rangle} \sqrt{\left\langle K^{*}, K^{*}\right\rangle}}=\frac{\left\langle K^{*}, K_{o}\right\rangle}{l \sqrt{\left\langle K^{*}, K^{*}\right\rangle}} .
$$

Given that

$$
\left\langle K^{*}, K_{o}\right\rangle=\left\langle\bar{K}+\tau Y V Y, K_{o}\right\rangle=\left\langle\bar{K}, K_{o}\right\rangle+\tau 1_{l}^{T} V 1_{l}=\left\langle\bar{K}, K_{o}\right\rangle+\tau S(V),
$$

and

$$
\left\langle K^{*}, K^{*}\right\rangle=\langle\bar{K}+\tau Y V Y, \bar{K}+\tau Y V Y\rangle=\langle\bar{K}, \bar{K}\rangle+2 \tau\langle\bar{K}, Y V Y\rangle+\tau^{2} S(V \otimes V),
$$

it holds that

$$
A\left(K^{*}, K_{o}\right)=\frac{\left\langle\bar{K}, K_{o}\right\rangle+\tau S(V)}{l \sqrt{\langle\bar{K}, \bar{K}\rangle+2 \tau\langle\bar{K}, Y V Y\rangle+\tau^{2} S(V \otimes V)}} .
$$


Since $S(V) \geq 0$ and $\tau \geq 0$ :

$$
\left\langle\bar{K}, K_{o}\right\rangle \leq l \sqrt{\langle\bar{K}, \bar{K}\rangle} ; \quad\left\langle\bar{K}, K_{o}\right\rangle[l \sqrt{\langle\bar{K}, \bar{K}\rangle}+\tau S(V)] \leq l \sqrt{\langle\bar{K}, \bar{K}\rangle}\left[\left\langle\bar{K}, K_{o}\right\rangle+\tau S(V)\right],
$$

and

$$
A\left(\bar{K}, K_{o}\right)=\frac{\left\langle\bar{K}, K_{o}\right\rangle}{l \sqrt{\langle\bar{K}, \bar{K}\rangle}} \leq \frac{\left\langle\bar{K}, K_{o}\right\rangle+\tau S(V)}{l\left[\sqrt{\langle\bar{K}, \bar{K}\rangle}+\frac{\tau}{l} S(V)\right]} .
$$

Now, using that $\tau \geq 0$ and the first assumption in Proposition 1,

$$
\begin{aligned}
\tau\left[S(V)^{2}-l^{2} S(V \otimes V)\right] & \geq 2 l^{2}\langle\bar{K}, Y V Y\rangle-2 S(V)\left\langle\bar{K}, K_{o}\right\rangle, \\
\frac{\tau^{2}}{l^{2}} S(V)^{2}+\frac{2 \tau}{l^{2}} S(V)\left\langle\bar{K}, K_{o}\right\rangle & \geq 2 \tau\langle\bar{K}, Y V Y\rangle+\tau^{2} S(V \otimes V), \\
\frac{\tau^{2}}{l^{2}} S(V)^{2}+\frac{2 \tau}{l} S(V) \sqrt{\langle\bar{K}, \bar{K}\rangle} & \geq 2 \tau\langle\bar{K}, Y V Y\rangle+\tau^{2} S(V \otimes V), \\
{\left[\sqrt{\langle\bar{K}, \bar{K}\rangle}+\frac{\tau}{l} S(V)\right]^{2} } & \geq\langle\bar{K}, \bar{K}\rangle+2 \tau\langle\bar{K}, Y V Y\rangle+\tau^{2} S(V \otimes V) .
\end{aligned}
$$

Using that $\tau \geq-\frac{\left\langle\bar{K}, K_{o}\right\rangle}{S(V)}$, it holds that

$$
\frac{\left\langle\bar{K}, K_{o}\right\rangle+\tau S(V)}{l\left[\sqrt{\langle\bar{K}, \bar{K}\rangle}+\frac{\tau}{l} S(V)\right]} \leq \frac{\left\langle\bar{K}, K_{o}\right\rangle+\tau S(V)}{l \sqrt{\langle\bar{K}, \bar{K}\rangle+2 \tau\langle\bar{K}, Y V Y\rangle+\tau^{2} S(V \otimes V)}}=A\left(K^{*}, K_{o}\right) .
$$

From (24) and (25) we have $A\left(K^{*}, K_{o}\right) \geq A\left(\bar{K}, K_{o}\right)$.

\section{References}

Alpayadin, E., \& Kaynak, C. (1998). Cascading classifiers. Kybernetika, 34(4), 369-374.

Bach, F., Lanckriet, G., \& Jordan, M. (2004). Multiple kernel learning, conic duality and the SMO algorithm. In Proceedings of the 21 st international conference machine learning. New York: ACM.

Bennett, K., Momma, M., \& Embrechts, J. (2002). MARK: a boosting algorithm for heterogeneous kernel models. In Proceedings of SIGKDD international conference on knowledge discovery and data mining.

Blake, C. L., \& Merz, C. J. (1998). UCI repository of machine learning databases. University of Carolina, Irvine, Department of Information and Computer Sciences, http://www.ics.uci.edu/ mlearn/ MLRepository.html.

Bousquet, O., \& Herrmann, D. (2003). On the complexity of learning the kernel matrix. In S. Becker, S. Thurn, \& K. Obermayer (Eds.), Advances in neural information processing systems: Vol. 15 (pp. 415422). Cambridge: MIT Press.

Burges, C. (1998). Geometry and invariance in kernel based methods. In Advances in kernel methodssupport vector learning (pp. 89-116). Cambridge: MIT Press.

Cortes, C., \& Vapnik, V. (1995). Support vector networks. Machine Learning, 20, 273-297.

Cox, T. F., \& Cox, M. A. A. (1994). Multidimensional scaling. London: Chapman and Hall.

Cristianini, N., \& Shawe-Taylor, J. (2000). Introduction to support vector machines. Cambridge: Cambridge University Press.

Cristianini, N., Shawe-Taylor, J., Elisseeff, A., \& Kandola, J. (2002). On kernel-target alignment. In T. Dietterich, S. Becker, \& Z. Ghahramani (Eds.), Advances in neural information processing systems: Vol. 14 (pp. 367-373). Cambridge: MIT Press. 
de Diego, M.I., Moguerza, J., \& Muñoz, A. (2004). Combining kernel information for support vector classification. In LNCS: Vol. 3077. Proceedings of the 5th international workshop MCS 2004 (pp. 102-111). Berlin: Springer.

Demsar, J. (2006). Statistical comparison of classifiers over multiple data sets. Journal of Machine Learning Research, 7, 1-20.

Domeniconi, F., Peng, J., \& Gunopulos, D. (2000). Adaptive metric nearest neighbor classification. In Proceedings of IEEE conference on CVPR (pp. 517-522).

Frey, P. W., \& Slate, D. J. (1991). Letter recognition using Holland-style adaptive classifiers. Machine Learning, 6(2), 161-182.

Fukushima, K., \& Imagawa, T. (1993). Recognition and segmentation of connected characters with selective attention. Neural Networks, 6, 33-41.

Gower, J. C., \& Legendre, P. (1986). Metric and euclidean properties of dissimilarity coefficients. Journal of Classification, 3, 5-48.

Haasdonk, B. (2005). Feature space interpretation of SVMs with indefinite kernels. IEEE Transactions on Pattern Analysis and Machine Intelligence, 27(4), 482-492.

Hastie, T., Tibshirani, R., \& Friedman, J. (2001). The elements of statistical learning. Berlin: Springer.

Jacobs, R. A., Jordan, M. I., Nowlan, S. J., \& Hinton, G. E. (1991). Adaptive mixture of local experts. Neural Computation, 3, 79-87.

Joachims, T. (2002). Learning to classify text using support vector machines. Norwell: Kluwer Academic.

Keerthi, S. S., \& Lin, C.-J. (2003). Asymptotic behaviors of support vector machines with Gaussian kernel. Neural Computation, 15, 1667-1689.

Kosko, B. (1991). Neural networks and fuzzy systems: a dynamical approach to machine intelligence. New York: Prentice Hall.

Kuncheva, L. I., \& Rodríguez, J. (2007). An experimental study on rotation forest ensembles. In LNCS: Vol. 4472. Proceedings of the 7th international workshop MCS 2007 (pp. 459-468). Berlin: Springer.

Kwok, J. T., \& Tsang, I. W. (2003). Learning with idealized kernels. In Proceedings of the 19th international conference machine learning (pp. 400-407).

Lanckriet, G. R. G., Cristianini, N., Barlett, P., El Ghaoui, L., \& Jordan, M. I. (2004). Learning the kernel matrix with semi-definite programming. Journal of Machine Learning Research, 5, 27-72.

Lehmann, E. L. (1975). Nonparametrics: statistical methods based on ranks. New Delhi: McGraw-Hill.

Mangasarian, O. L., \& Wolberg, W. H. (1990). Cancer diagnosis via linear programming. SIAM News, 23(5), $1-18$.

McLachlan, G., \& Peel, D. (2000). Finite mixture models. New York: Wiley.

Moguerza, J. M., Muñoz, A., \& Martín Merino, M. (2002). Detecting the number of clusters using a support vector machine approach. In LNCS: Vol. 2415. Proceedings of the ICANN 2002 (pp. 763-768). Berlin: Springer.

Moguerza, J. M., de Diego, M. I., \& Muñoz, A. (2004). Improving support vector classification via the combination of multiple sources of information. In LNCS: Vol. 3138. Proceedings of the IAPR international workshops SSPR 2004 and SPR 2004 (pp. 592-600). Berlin: Springer.

Muñoz, A. (1997). Compound key word generation from document databases using a hierarchical clustering ART model. Intelligent Data Analysis, 1, 25-48.

Muñoz, A., \& Moguerza, J. M. (2006). Estimation of high-density regions using one-class neighbor machines. IEEE Transactions on Pattern Analysis and Machine Intelligence, 28(3), 476-480.

Muñoz, A., \& Villagarcía, T. (1998). Unsupervised neural networks for variable selection with mixed covariates. In Analyse multidimensionelle des donnes, CSIA Ceresta (pp. 217-227).

Ong, C. S., Mary, X., Canu, S., \& Smola, A. (2004). Learning with non-positive kernels. In Proceedings of the 21st international conference on machine learning (pp. 639-646). Berlin: Springer.

Ong, C. S., Smola, A., \& Williamson, R. (2005). Learning the kernel with Hyperkernels. Journal of Machine Learning Research, 6, 1043-1071.

Pȩkalska, E., \& Duin, R. P. W. (2005). The dissimilarity representation for pattern recognition. Foundations and applications. Singapore: World Scientific.

Pȩkalska, E., Paclík, P., \& Duin, R. P. W. (2001). A generalized kernel approach to dissimilarity-based classification. Journal of Machine Learning Research, Special Issue on Kernel Methods, 2(12), 175-211.

Pȩkalska, E., Duin, R. P. W., Günter, S., \& Bunke, H. (2004). On not making dissimilarities euclidean. In LNCS: Vol. 3138. Proceedings of the IAPR international workshops SSPR 2004 and SPR 2004 (pp. 1145-1154). Berlin: Springer.

Schölkopf, B., \& Smola, A. (2002). Learning with kernels. Cambridge: MIT Press.

Schölkopf, B., Mika, S., Burges, C. J., P. Knirsch, K.-R. M., Rätsch, G., \& Smola, A. J. (1999). Input space vs. feature space in kernel-based methods. IEEE Transactions on Neural Networks.

Schölkopf, B., Herbrich, R., Smola, A., \& Williamson, R. (2000). A generalized representer theorem, Tech. Rep. No. NC2-TR-2000-81, NeuroCOLT2. 
Shawe-Taylor, J., \& Cristianini, N. (2004). Kernel methods for pattern analysis. Cambridge: Cambridge University Press.

Silverman, B. (1986). Density estimation for statistics and data analysis. London: Chapman and Hall.

Tsochantaridis, I., Hofmann, T., Joachims, T., \& Altun, Y. (2004). Support vector machine learning for interdependent and structured output spaces. In Proceedings of the 21st international conference machine learning (pp. 823-830). New York: ACM Press.

Tsuda, K. (1999). Support vector classifier with asymmetric kernel functions. In Proceedings of the European symposium of artificial neural networks, ESANN'99 (pp. 183-188).

Vandenberghe, L., \& Boyd, S. (1996). Semidefinite programming. SIAM Review, 38(1), 49-95.

Zielman, B., \& Heiser, W. J. (1996). Models for asymmetric proximities. British Journal of Mathematical and Statistical Psychology, 49, 127-146. 